\title{
Metric properties of Cayley graphs of alternating groups
}

\section{Olshevskyi M.S.}

\begin{abstract}
A well known diameter search problem for finite groups with respect to its systems of generators is considered. The problem can be formulated as follows: find the diameter of a group over its system of generators. The diameter of a group over a specific system of generators is the diameter of the corresponding Cayley graph.

It is considered alternating groups with classic irreducible system of generators consisting of cycles with length three of the form $(1,2, k)$. The main part of the paper concentrates on analysis how even permutations decompose with respect to this system of generators. The rules for moving generators from permutation's decomposition from left to right and from right to left are introduced. These rules give rise for transformations of decompositions, that do not increase their lengths. They are applied for removing fixed points of a permutation, that were included in its decomposition. Based on this rule the stability of system of generators is proved. The strict growing property of the system of generators is also proved, as the corollary of transformation rules and the stability property.

It is considered homogeneous theory, that was introduced in the previous author's paper. For the series of alternating groups with systems of generators mentioned above it is shown that this series is uniform and homogeneous. It makes possible to apply the homogeneous down search algorithm to compute the diameter. This algorithm is applied and exact values of diameters for alternating groups of degree up to 43 are computed.

Key words and phrases: Cayley graph, graph diameter, system of generators, alternating group.
\end{abstract}

Taras Shevchenko National University, 64/13 Volodymyrska str., 01601, Kyiv, Ukraine

E-mail: msolshevskyi@gmail.com

\section{Introduction}

In group theory the diameter search problem for specific system of generators can be formulated as follows. For a finite group $G$ and its system of generators $S$ find the diameter $D_{S}(G)$ of the Cayley graph $\Gamma(G, S)$ of $G$ with respect to $S$. The diameter of $\operatorname{Sym}(n)$ over $S=\{(1, k): k \in 2, \ldots, n\}$ was found in [1].

The general variant of this problem, the diameter search problem, is formulated as the problem to find maximum of $D_{S}(G)$ for all systems of generators $S$ of $G$. The research in this direction was stimulated by the paper of L. Babai and A. Seress [2]. A few recent papers dealing with this topic are $[3,4,6,7,9]$.

The minimum-length generators sequence search problem is another well known problem for finite groups. It can be formulated as follows: for a finite group $G$, its system of generators $S$ and an element $g \in G$ find the shortest generators sequence realizing $g$. In [5], it is shown that this problem is NP-hard for permutation groups. 
In the present work, we consider alternating groups $\operatorname{Alt}(n), n \geq 3$. As a system of generators of $A l t(n)$ we consider $\operatorname{So} G(n)$ consisting of cycles $(1,2, k), k=3, \ldots, n$. We investigate properties of multiplication of even permutations by such generators. These properties provide an opportunity to establish rules of moving generators in permutation's decompositions from left to right and from right to left. This leads to the proof of the main result of the paper. We show that our groups with respect to mentioned systems of generators are stable, i.e. for an even permutation its minimal length of a decomposition is preserved when the degree of the alternating group grows. Also, we show that our system of generators is strictly growing.

This paper is partially based on the previous author's work [8]. We consider the series of alternating groups and their generator systems and show that it is uniform and homogeneous. Then to find the diameter we apply the homogeneous down search algorithm. As a result of computations we present the exact values of diameters for alternating groups $\operatorname{Alt}(n)$ with respect to generators $(1,2, k), k=3, \ldots, n$, of degree $n$ up to 43 .

The paper is organized as follows. Section 1 describes specific notations, elementary properties of generators and introduces one of the main tools of the paper, the trajectory of a point over permutation's decomposition. In Section 2, the rules of moving generators over decompositions from left to right or from right to left are obtained. In Section 3, using the rules from previous section it is proved stability of alternating groups with respect to considered systems of generators. Also, as corollary it is shown that the strictly growing property of these systems of generators hold. Section 4 recalls definitions of groups-generators series. It is proved that alternating groups-generators series is uniform and homogeneous. Homogeneous down search algorithm is applied for the alternating groups of degrees up to 43.

Unless otherwise specified in the paper we denote a finite group by $G$ and a system of generators of $G$ by $S$.

\section{Preliminaries}

The main object of research of the paper is the alternating group Alt $n)$ with its system of generators $((1,2,3), \ldots,(1,2, n)), n \geq 3$. We consider some useful elementary properties of this system of generators and introduce the trajectory of a point over permutation's decomposition. This notion will be intensively used in the sequel and we present general classification of trajectories.

\subsection{Basic definitions, notations and properties}

Every element $g$ of $G$ can be decomposed as a product

$$
g=\prod_{k=1}^{l} s_{k}
$$

of generators from $S$ for some natural $l$. The tuple of generators $\left(s_{1}, \ldots, s_{k}\right)$ will be called a decomposition of the element $g$ over $S$. The length $|g|_{S}$ of the element $g$ over $S$ is the length of the shortest decomposition of $g$ over $S$. The diameter $D_{S}(G)$ of $G$ with respect to $S$ is the maximum of lengths $|g|_{S}, g \in G$. An element $g \in G$ such that $|g|_{S}=D_{S}(G)$ is called a diameter element.

Let us introduce the operation of concatenation over tuples of generators. Let $\pi, \tau$ be elements from $G$ and $D=\left(d_{1}, \ldots, d_{m}\right), T=\left(t_{1}, \ldots, t_{u}\right)$ be its decompositions over $S$ correspondingly. Then the concatenation of $D$ and $T$ is the decomposition $\left(d_{1}, \ldots, d_{m}, t_{1}, \ldots, t_{u}\right)$ of the element $\pi \cdot \tau$. 
An element $a \in G$ will be called properly generated over $S$ if for arbitrary $A \subset S, A \neq S$, we have $a \notin\langle A\rangle$. A system of generators $S$ of a group $G$ will be called strictly growing if every diameter element is properly generated.

Fix an integer $n_{0}$.

Definition 1. A groups-generators series $\mathbb{G}$ is the sequence of pairs $\left(G(n)\right.$, SoG $\left.(n): n>n_{0}\right)$ such that:

1) $G\left(n_{0}+1\right)<G\left(n_{0}+2\right)<G\left(n_{0}+3\right)<\ldots$ is an ascending group series;

2) $\operatorname{So} G(n)$ is a system of generators of $G(n)$ and $\operatorname{So} G(n) \subset \operatorname{SoG}(n+1), n>n_{0}$.

Let $\mathrm{G}$ be a groups-generators series.

Definition 2. The series $\mathbb{G}$ will be called stable if for arbitrary integers $N_{1}, N_{2}, n_{0}<N_{1}<N_{2}$, and every element $a \in G\left(N_{1}\right)$ the following equality holds

$$
|a|_{\operatorname{SoG}\left(N_{1}\right)}=|a|_{\operatorname{SoG}\left(N_{2}\right)} \text {. }
$$

Let $G(n)$ be the alternating group $\operatorname{Alt}(n), n \geq 3$. We fix the natural embedding of $\operatorname{Alt}(n)$ into $\operatorname{Alt}(n+1), n \geq 3$. Denote by $s_{k}$ the cycle $(1,2, k), k \geq 3$. Let $\left.\operatorname{So} G(n)=\left\{s_{3}, \ldots, s_{n}\right)\right\}, n \geq 3$. Denote by $\mathbb{A}$ the groups-generators series $((\operatorname{Alt}(n), \operatorname{So} G(n)): n \geq 3)$.

In this paper, we consider the right rule of permutation's multiplication: for every permutations $\pi, \tau$ and for every natural number $x$ we have

$$
(\pi \cdot \tau)(x)=\tau(\pi(x))
$$

As usual, the support of a permutation $\pi$ in $A$ will be denoted by $\operatorname{supp}(\pi)$, i.e. $\operatorname{supp}(\pi)=$ $\{x \in \overline{1, n}: \pi(x) \neq x\}$, where $\overline{1, n}=\{1,2, \ldots, n\}$.

Fix a natural number $n \geq 3$. The group $A=\operatorname{Alt}(n)$ is a permutation group on the set $\overline{1, n}$. The elements of this set will be called points.

Let $S=\operatorname{SoG}(n)$. Let $D=\left(i_{1}, \ldots, i_{m}\right)$ be a tuple of $m \geq 1$ natural numbers, each greater or equal than 3 . We will use notation $\left[i_{1}, \ldots, i_{m}\right]_{S}$ for the product

$$
\prod_{k=1}^{m} s_{i_{k}}=\prod_{k=1}^{m}\left(1,2, i_{k}\right) .
$$

For simplicity we will identify the tuple $D=\left(i_{1}, \ldots, i_{m}\right)$ of indices with the tuple $\left(s_{i_{1}}, \ldots, s_{i_{m}}\right)$ of generators. The later forms a decomposition of the permutation $\left[i_{1}, \ldots, i_{m}\right]_{S}$ over $S$.

We will use notation $\overbrace{a \rightarrow b}^{\pi}$ for $\pi \in A$ and $a, b \in \overline{1, n}$ such that $\pi(a)=b$. Moreover, if for some permutation $\tau \in A$ and a point $c$ additional equality $\tau(b)=c$ holds, then we use the following notation $\overbrace{a \rightarrow b}^{\pi} \Rightarrow \overbrace{b \rightarrow c}^{\tau}$. This notation can be naturally generalized for products of arbitrary number of permutations.

Proposition 1. The following equalities hold:

1) $[i, i, i]_{S}=e, i \geq 3$;

2) $[i, j]_{S}=[j, j, i, i]_{S}, i, j \geq 3, i \neq j$. 
Proof. 1. Every cycle of length $t$ has order $t$. Then $[i, i, i]_{S}=(1,2, i)^{3}=e$.

2. It is enough to show that $s_{i} \cdot s_{j}$ and $s_{j} \cdot s_{j} \cdot s_{i} \cdot s_{i}$ coincide on every point from the set $\{1,2, i, j\}$. Direct computations are summarized in the following table.

\begin{tabular}{|c|c|c|}
\hline Case & $\overbrace{1 \rightarrow 2}^{s_{i} \cdot s_{j}} \Rightarrow \overbrace{2 \rightarrow j}^{s_{j}}$ & $\overbrace{1 \rightarrow 2}^{s_{j}} \Rightarrow \overbrace{2 \rightarrow j}^{s_{j}} \Rightarrow \overbrace{j \rightarrow j}^{s_{j}} \Rightarrow \overbrace{j \rightarrow j}^{s_{j}} \cdot s_{i} \cdot s_{i}$ \\
\hline 2 & $\overbrace{2 \rightarrow i}^{s_{i}} \Rightarrow \overbrace{i \rightarrow i}^{s_{j}}$ & $\overbrace{2 \rightarrow j}^{s_{j}} \Rightarrow \overbrace{j \rightarrow 1}^{s_{j}} \Rightarrow \overbrace{1 \rightarrow 2}^{s_{i}} \Rightarrow \overbrace{2 \rightarrow i}^{s_{i}}$ \\
\hline$i$ & $\overbrace{i \rightarrow 1}^{s_{i}} \Rightarrow \overbrace{1 \rightarrow 2}^{s_{j}}$ & $\overbrace{i \rightarrow i}^{s_{j}} \Rightarrow \overbrace{i \rightarrow i}^{s_{j}} \Rightarrow \overbrace{i \rightarrow 1}^{s_{i}} \Rightarrow \overbrace{1 \rightarrow 2}^{s_{i}}$ \\
\hline$j$ & $\overbrace{j \rightarrow j}^{s_{i}} \Rightarrow \overbrace{j \rightarrow 1}^{s_{j}}$ & $\overbrace{j \rightarrow 1}^{s_{j}} \Rightarrow \overbrace{1 \rightarrow 2}^{s_{j}} \Rightarrow \overbrace{2 \rightarrow i}^{s_{i}} \Rightarrow \overbrace{i \rightarrow 1}^{s_{i}}$ \\
\hline
\end{tabular}

\subsection{The trajectory of a point over a decomposition}

Let $\pi \in A$ be a permutation. Fix a decomposition $D=\left(i_{1}, \ldots, i_{m}\right)$ of $\pi$ over $S$. We assume that $m \geq 3$. Let $a \in\left\{1,2, i_{1}, \ldots, i_{m}\right\}$.

We will define the trajectory of $a$ over the decomposition $D$ as follows. For arbitrary points $c, d$ and $k \in \overline{1, m}$ such that $s_{i_{k}}(c)=d$ we will use notation $c \stackrel{k}{\rightarrow} d$. Then in the graph of the action of the generating system $S$ on $\overline{1, n}$ the decomposition $D$ defines the unique path of the form

$$
a=a_{0} \stackrel{1}{\rightarrow} a_{1} \stackrel{2}{\rightarrow} a_{2} \stackrel{3}{\rightarrow} \ldots \stackrel{m}{\rightarrow} a_{m}=\pi(a)
$$

Definition 3. The trajectory of the point $a$ in the permutation $\pi$ over the decomposition $D$ is a tuple $\operatorname{Tr}(a, \pi, D):=\left(j_{1}, \ldots, j_{t}\right)$ of all indices from $D$, such that exactly one condition $a_{j_{k}}=1$ or $a_{j_{k}-1}=2$ holds for every $1 \leq k \leq t$.

In other words, the trajectory of $a$ is the tuple of all positions in $D$, such that in the graph of the action corresponding generator defines the arrow that either starts in 2 or terminates at 1. Since $m \geq 3$ the trajectory is well defined.

In many cases we can use more compact form of the path of a point. For arbitrary $k, l \in \overline{1, m}$, $k<l$, such that $2=s_{i_{k}}^{-1}\left(a_{k}\right) \neq a_{k}=\ldots=a_{l-1} \neq s_{i_{l}}\left(a_{l-1}\right)=1, a_{k} \neq 1,2$, we will use the notation $a_{k} \stackrel{k, l}{\Rightarrow} a_{k}$. Note that in this case both $k, l \in \operatorname{Tr}(a, \pi, D)$.

The following properties of the trajectory $\operatorname{Tr}(a, \pi, D)=\left(j_{1}, \ldots, j_{t}\right)$ hold:

1) $a_{j_{k}} \neq 2$ for every $k \in \overline{1, t}$;

2) $a_{j_{k}}=a_{j_{k+1}-1}$ if and only if $a_{j_{k}} \stackrel{j_{k} j_{k+1}}{\longrightarrow} a_{j_{k}}$ is well defined in the path of $a$ over $D$.

We will omit the following parts of the path of $a$ in $\pi$ over $D$ :

1) the initial part of the path up to the first occurrence of $a$, if $a \neq 1,2$;

2) the closing part of the path after the last occurrence of $\pi(a)$, if $\pi(a) \neq 1,2$.

Under these conditions we have the following assertion. 
Proposition 2. Let $\pi \in A, D$ be its decomposition over $S$ and $a$ be a point from $\{1,2\} \cup D$. Depending on the values of $a$ and $\pi(a)$ the path of $a$ over $D$ has one the following forms.

Case 1.1. $a=1, \pi(a)=1$. Then

$1 \stackrel{1}{\rightarrow} 2 \stackrel{2}{\rightarrow} i_{j_{1}} \stackrel{\stackrel{j_{1}, j_{2}}{\longrightarrow}}{\longrightarrow} i_{j_{2}} \stackrel{j_{2}}{\longrightarrow} 1 \stackrel{j_{2}+1}{\longrightarrow} 2 \stackrel{j_{2}+2}{\longrightarrow} i_{j_{3}} \stackrel{j_{3, j_{4}}}{\longrightarrow} \ldots i_{j_{t-2}} \stackrel{j_{t-2}}{\longrightarrow} 1 \stackrel{j_{t-2}+1}{\longrightarrow} 2 \stackrel{j_{t-2}+2}{\longrightarrow} i_{j_{t-1}} \stackrel{j_{t-1, j_{t}}}{\longrightarrow} i_{j_{t}} \stackrel{m}{\longrightarrow} 1$

Case 1.2. $a=1, \pi(a)=2$. Then

$$
\begin{gathered}
1 \stackrel{1}{\rightarrow} 2 \stackrel{2}{\rightarrow} i_{j_{1}} \stackrel{j_{1}, j_{2}}{\longrightarrow} i_{j_{2}} \stackrel{j_{2}}{\longrightarrow} 1 \stackrel{j_{2}+1}{\longrightarrow} 2 \stackrel{j_{2}+2}{\longrightarrow} i_{j_{3}} \stackrel{j_{3, j_{4}}}{\longrightarrow} \\
i_{j_{t-2}} \stackrel{\ldots \ldots \ldots}{\longrightarrow} 1 \stackrel{j_{t-2}}{\longrightarrow} \stackrel{j_{t-2}+1}{\longrightarrow} 2 \stackrel{j_{t-2}+2}{\longrightarrow} i_{j_{t-1}} \stackrel{j_{t-1, j_{t}}}{\longrightarrow} i_{j_{t}} \stackrel{m-1}{\longrightarrow} 1 \stackrel{m}{\longrightarrow} 2
\end{gathered}
$$

Case 1.3. $a=1, \pi(a) \neq 1,2$. Then

$$
\begin{aligned}
& 1 \stackrel{1}{\rightarrow} 2 \stackrel{2}{\rightarrow} i_{j_{1}} \stackrel{j_{1}, j_{2}}{\longrightarrow} i_{j_{2}} \stackrel{j_{2}}{\longrightarrow} 1 \stackrel{j_{2}+1}{\longrightarrow} 2 \stackrel{j_{2}+2}{\longrightarrow} i_{j_{3}} \stackrel{j_{3}, j_{4}}{\longrightarrow} \\
& i_{j_{t-3}} \stackrel{j_{t-3}}{\longrightarrow} 1 \stackrel{j_{t-3}+1}{\longrightarrow} 2 \stackrel{j_{t-3}+2}{\longrightarrow} i_{j_{t-2}} \stackrel{j_{t-2}, j_{t-1}}{\longrightarrow} i_{j_{t-1}} \stackrel{j_{t-1}}{\longrightarrow} 1 \stackrel{j_{t-1}+1}{\longrightarrow} 2 \stackrel{j_{t-1}+2}{\longrightarrow} i_{j_{t}}
\end{aligned}
$$

Case 2.1. $a=2, \pi(a)=1$. Then

$2 \stackrel{1}{\rightarrow} i_{j_{1}} \stackrel{j_{1} j_{2}}{\longrightarrow} i_{j_{2}} \stackrel{j_{2}}{\longrightarrow} 1 \stackrel{j_{2}+1}{\longrightarrow} 2 \stackrel{j_{2}+2}{\longrightarrow} i_{j_{3}} \stackrel{j_{3}, j_{4}}{\longrightarrow} \ldots i_{j_{t-2}} \stackrel{j_{t-2}}{\longrightarrow} 1 \stackrel{j_{t-2}+1}{\longrightarrow} 2 \stackrel{j_{t-2}+2}{\longrightarrow} i_{j_{t-1}} \stackrel{j_{t-1, j_{t}}}{\longrightarrow} i_{j_{t}} \stackrel{m}{\longrightarrow} 1$

Case 2.2. $a=2, \pi(a)=2$. Then

$2 \stackrel{1}{\rightarrow} i_{j_{1}} \stackrel{j_{1}, j_{2}}{\longrightarrow} i_{j_{2}} \stackrel{j_{2}}{\longrightarrow} 1 \stackrel{j_{2}+1}{\longrightarrow} 2 \stackrel{j_{2}+2}{\longrightarrow} i_{j_{3}} \stackrel{j_{3} j_{4}}{\longrightarrow} \ldots i_{j_{t-2}} \stackrel{j_{t-2}}{\longrightarrow} 1 \stackrel{j_{t-2}+1}{\longrightarrow} 2 \stackrel{j_{t-2}+2}{\longrightarrow} i_{j_{t-1}} \stackrel{j_{t-1}, j_{t}}{\longrightarrow} i_{j_{t}} \stackrel{m-1}{\longrightarrow} 1 \stackrel{m}{\longrightarrow} 2$

Case 2.3. $a=2, \pi(a) \neq 1,2$. Then

$$
\begin{aligned}
& 2 \stackrel{1}{\rightarrow} i_{j_{1}} \stackrel{j_{1}, j_{2}}{\longrightarrow} i_{j_{2}} \stackrel{j_{2}}{\longrightarrow} 1 \stackrel{j_{2}+1}{\longrightarrow} 2 \stackrel{j_{2}+2}{\longrightarrow} i_{j_{3}} \stackrel{j_{3} j_{4}}{\longrightarrow} \\
& i_{j_{t-3}} \stackrel{j_{t-3}}{\longrightarrow} 1 \stackrel{j_{t-3}+1}{\longrightarrow} 2 \stackrel{j_{t-3}+2}{\longrightarrow} i_{j_{t-2}} \stackrel{j_{t-2}, j_{t-1}}{\longrightarrow} i_{j_{t-1}} \stackrel{j_{t-1}}{\longrightarrow} 1 \stackrel{j_{t-1}+1}{\longrightarrow} 2 \stackrel{j_{t-1}+2}{\longrightarrow} i_{j_{t}}
\end{aligned}
$$

Case 3.1. $a \neq 1,2, \pi(a)=1$. Then

$$
\begin{gathered}
i_{j_{1}} \stackrel{j_{1}}{\longrightarrow} 1 \stackrel{j_{1}+1}{\longrightarrow} 2 \stackrel{j_{1}+2}{\longrightarrow} i_{j_{2}} \stackrel{j_{2}, j_{3}}{\longrightarrow} i_{j_{3}} \stackrel{j_{3}}{\longrightarrow} 1 \stackrel{j_{3}+1}{\longrightarrow} 2 \stackrel{j_{3}+2}{\longrightarrow} i_{j_{4}} \stackrel{j_{4}, j_{5}}{\longrightarrow} \\
\ldots \ldots \ldots \ldots \\
i_{j_{t-2}} \stackrel{j_{t-2}}{\longrightarrow} 1 \stackrel{j_{t-2}+1}{\longrightarrow} 2 \stackrel{j_{t-2}+2}{\longrightarrow} i_{j_{t-1}} \stackrel{j_{t-1, j_{t}}}{\longrightarrow} i_{j_{t}} \stackrel{m}{\longrightarrow} 1
\end{gathered}
$$

Case 3.2. $a \neq 1,2, \pi(a)=2$. Then

$$
\begin{gathered}
i_{j_{1}} \stackrel{j_{1}}{\longrightarrow} 1 \stackrel{j_{1}+1}{\longrightarrow} 2 \stackrel{j_{1}+2}{\longrightarrow} i_{j_{2}} \stackrel{j_{2}, j_{3}}{\longrightarrow} i_{j_{3}} \stackrel{j_{3}}{\longrightarrow} 1 \stackrel{j_{3}+1}{\longrightarrow} 2 \stackrel{j_{3}+2}{\longrightarrow} i_{j_{4}} \stackrel{j_{4}, j_{5}}{\longrightarrow} \\
\ldots \ldots \ldots \ldots \ldots \\
i_{j_{t-2}} \stackrel{j_{t-2}}{\longrightarrow} 1 \stackrel{j_{t-2}+1}{\longrightarrow} 2 \stackrel{j_{t-2}+2}{\longrightarrow} i_{j_{t-1}} \stackrel{j_{t-1, j_{t}}}{\longrightarrow} i_{j_{t}} \stackrel{m-1}{\longrightarrow} 1 \stackrel{m}{\longrightarrow} 2
\end{gathered}
$$

Case 3.3. $\neq 1,2, \pi(a) \neq 1,2$. Then

$$
\begin{aligned}
& i_{j_{1}} \stackrel{j_{1}}{\longrightarrow} 1 \stackrel{j_{1}+1}{\longrightarrow} 2 \stackrel{j_{1}+2}{\longrightarrow} i_{j_{2}} \stackrel{j_{2}, j_{3}}{\longrightarrow} i_{j_{3}} \stackrel{j_{3}}{\longrightarrow} 1 \stackrel{j_{3}+1}{\longrightarrow} 2 \stackrel{j_{3}+2}{\longrightarrow} i_{j_{4}} \stackrel{j_{4}, j_{5}}{\longrightarrow} \\
& i_{j_{t-3}} \stackrel{j_{t-3}}{\longrightarrow} 1 \stackrel{j_{t-3}+1}{\longrightarrow} 2 \stackrel{j_{t-3}+2}{\longrightarrow} i_{j_{t-2}} \stackrel{j_{t-2}, j_{t-1}}{\longrightarrow} i_{j_{t-1}} \stackrel{j_{t-1}}{\longrightarrow} 1 \stackrel{j_{t-1}+1}{\longrightarrow} 2 \stackrel{j_{t-1}+2}{\longrightarrow} i_{j_{t}}
\end{aligned}
$$

Let $\pi \in A$ be a permutation with decomposition $D=\left(d_{1}, \ldots, d_{m}\right)$ over $S, m \geq 3$, and $p$ be a point from $\left\{1,2, d_{1}, \ldots, d_{m}\right\}$. We will say that the point $p$ has trivial path in $\pi$ over $D$ if $|\operatorname{Tr}(p, \pi, D)| \leq 2$. 


\section{Multiplication of permutations by generators}

In order to prove stability of the series $\mathbb{A}$ we need to establish how a decomposition of a permutations from $A$ behaviors being multiplied by a generator element from $S$. More precisely, in this section we show that conjugation by a generator or its inverse does not increase the length of a decomposition. The proof breaks into a series of lemmas.

Lemma 1. Let $\pi \in A, D=\left(d_{1}, \ldots, d_{m}\right), m \geq 3$, be its decomposition over $S, d$ be some point from $D$. Then for arbitrary point $p$ such that $p \geq 3$ and $p \neq d_{k}, k \in \overline{1, m}$, permutation $\tau \in A$, defined by the equality

$$
\tau=\left[t_{1}, \ldots, t_{m}\right], \quad \text { where } t_{i}= \begin{cases}p, & \text { if } d_{i}=d, \\ d_{i}, & \text { otherwise, }\end{cases}
$$

and each point $x$ the following equality holds

$$
\tau(x)= \begin{cases}p, & \text { if } \pi(x)=d \\ \pi(p), & \text { if } x=d \\ \pi(x), & \text { otherwise. }\end{cases}
$$

Proof. The decomposition $T=\left(t_{1}, \ldots, t_{m}\right)$ of $\tau$ is obtained from $D$ using substitutions of $d$ by $p$. Note that in the graph of the action on a point $x$ this transformation changes only one vertex. Namely, the vertex $d$ substituted by $p$. Hence, paths of $x$, which are defined by decompositions of $\pi$ and $\tau$, have the same labels on arrows. As the result, for every point $x, x \neq d$ and $\pi(x) \neq d$, we have $\operatorname{Tr}(x, \pi, D)=\operatorname{Tr}(x, \tau, T)$. This equality implies that $\tau(x)=\pi(x)$ for every $x \in \operatorname{supp}(\pi), \pi(x) \neq d$.

Moreover, equality (1) implies:

1) from $\operatorname{Tr}(d, \pi, D)=\operatorname{Tr}(p, \tau, T)$ it follows that:

(a) if $\pi(d)=d$, then $\tau(p)=p$,

(b) if $\pi(d) \neq d$, then $\tau(p)=\pi(d)$;

2) from $\pi(p)=p$ it follows that $\tau(d)=d$.

This completes the proof.

Lemma 2. Let $\pi \in A$ be a permutation such that 1,2 $\notin \operatorname{supp}(\pi)$. Then for each point $p$ such that $p \geq 3$ and $p \notin \operatorname{supp}(\pi)$ the equality $s_{p} \cdot \pi=\pi \cdot s_{p}$ holds.

Proof. Note, that $\operatorname{supp}\left(s_{p}\right) \cap \operatorname{supp}(\pi)=\varnothing$. It is enough to show, that $\left(s_{p} \cdot \pi\right)(x)=\left(\pi \cdot s_{p}\right)(x)$ for every point $x \in\{1,2, p\} \bigcup \operatorname{supp}(\pi)$. The results of direct computations are summarized in the following table.

\begin{tabular}{|c|c|c|}
\hline Case & $\overbrace{1 \rightarrow 2}^{s_{p} \cdot \pi} \Rightarrow \overbrace{2 \rightarrow 2}^{s_{p}}$ & $\overbrace{1 \rightarrow 1}^{\pi} \Rightarrow \overbrace{1 \rightarrow 2}^{s_{p}}$ \\
\hline$x=1$ & $\overbrace{2 \rightarrow p}^{s_{p}} \Rightarrow \overbrace{p \rightarrow p}^{\pi}$ & $\overbrace{2 \rightarrow 2}^{\pi} \Rightarrow \overbrace{2 \rightarrow p}^{s_{p}}$ \\
\hline$x=2$ & $\overbrace{p \rightarrow 1}^{s_{p}} \Rightarrow \overbrace{1 \rightarrow 1}^{\pi}$ & $\overbrace{p \rightarrow p}^{\pi} \Rightarrow \overbrace{p \rightarrow 1}^{s_{p}}$ \\
\hline$x \in \operatorname{supp}(\pi)$ & $\overbrace{x \rightarrow x}^{s_{p}} \Rightarrow \overbrace{x \rightarrow \pi(x)}^{\pi}$ & $\overbrace{x \rightarrow \pi(x)}^{\pi} \Rightarrow \overbrace{\pi(x) \rightarrow \pi(x)}^{s_{p}}$ \\
\hline
\end{tabular}

Hence, $s_{p} \cdot \pi=\pi \cdot s_{p}$. 


\subsection{Multiplication from the left}

Let $\pi \in A$ be a permutation with decomposition $D=\left(d_{1}, \ldots, d_{m}\right)$ over $S, m \geq 3$. Note that if the point 1 is fixed in $\pi$ and has trivial path in $\pi$ over $D$, then via Proposition 2 the path of the point 1 over $D$ has the form

$$
1 \stackrel{1}{\rightarrow} 2 \stackrel{2}{\rightarrow} d_{2} \stackrel{d_{2}, d_{m}}{\longrightarrow} d_{m} \stackrel{d_{m}}{\longrightarrow} 1
$$

Lemma 3. Let $\pi \in A$ be a permutation with decomposition $D=\left(d_{1}, \ldots, d_{m}\right), m \geq 3,1$ is fixed in $\pi$ over $D$ with trivial path, $d=\pi^{-1}\left(d_{2}\right)$ and $p$ be a natural number $\geq 3$, such that there is no $k \in \overline{1, m}$ such that $p=d_{k}$. Then $s_{p} \cdot \pi=\tau \cdot s_{p}$, where

$$
\tau= \begin{cases}{\left[d, d_{1}, d_{2}, \ldots, d_{m-1}\right]_{s},} & \text { if } d \neq 2 \\ {\left[d_{1}, d_{2}, \ldots, d_{m-1}\right]_{s},} & \text { if } d=2\end{cases}
$$

Proof. Denote $\pi^{-1}(2)$ by $y$. Consider cases $d \neq 2$ and $d=2$ independently.

Let $d \neq 2$. Denote $\left[d_{1}, \ldots, d_{m-1}\right]_{S}$ by $\sigma$. From the construction of $\tau$ it follows that for every point $x, x \notin\{1,2, p, d, y\}$, we have $\pi(x) \notin\{1,2, p, d, y\}$. Therefore $\left(s_{p} \cdot \pi\right)(x)=\pi(x)=$ $\tau(x)=\left(\tau \cdot s_{p}\right)(x)$. Hence, it is enough to show that $\left(s_{p} \cdot \pi\right)(x)=\left(\tau \cdot s_{p}\right)(x)$ for every point $x$ from $\{1,2, p, d, y\}$.

Case $x=1$. Note, that $\pi(2) \neq 1$. So, $\pi(2)$ is some number from $\left\{d_{1}, d_{3}, \ldots, d_{m-1}\right\}$ or 2 . Consider cases:

1) if $\pi(2) \neq 2$, then $\pi(2)=\sigma(2)$. So, $\tau(1)=\pi(2)$;

2) if $\pi(2)=2$, then $\sigma(2)=1$. So, $\tau(1)=1$.

As the result, we have the following table.

\begin{tabular}{|l|c|c|}
\hline & $s_{p} \cdot \pi$ & $\tau \cdot s_{p}$ \\
\hline$\pi(2) \neq 2$ & $\overbrace{1 \rightarrow 2}^{s_{p}} \Rightarrow \overbrace{2 \rightarrow \pi(2)}^{\pi}$ & $\overbrace{1 \rightarrow \pi(2)}^{\tau} \Rightarrow \overbrace{\pi(2) \rightarrow \pi(2)}^{s_{p}}$ \\
$\pi(2)=2$ & $\overbrace{1 \rightarrow 2}^{s_{p}} \Rightarrow \overbrace{2 \rightarrow 2}^{\pi}$ & $\overbrace{1 \rightarrow 1}^{\tau} \Rightarrow \overbrace{1 \rightarrow 2}^{s_{p}}$ \\
\hline
\end{tabular}

Case $x=2$. Note, that from $\pi(d)=d_{m}$ it follows that the path of $d$ over $D$ ends on the $m$ th position. So, $\sigma(d)=2$, as $\sigma$ does not contain the $m$ th position of $D$. Hence, $\tau(2)=2$. As the result, we have the following table.

\begin{tabular}{|c|c|}
\hline$\overbrace{2 \rightarrow p}^{s_{p} \cdot \pi} \Rightarrow \overbrace{p \rightarrow p}^{s_{p}}$ & $\tau \cdot s_{p}$ \\
\hline$\overbrace{2 \rightarrow 2}^{\tau} \Rightarrow \overbrace{2 \rightarrow p}^{s_{p}}$ \\
\hline
\end{tabular}

Case $x=p$. In this case, we have the following table.

\begin{tabular}{|c|c|}
\hline$\overbrace{p \rightarrow 1}^{s_{p} \cdot \pi} \Rightarrow \overbrace{1 \rightarrow 1}^{s_{p}}$ & $\tau \cdot s_{p}$ \\
\hline & $\overbrace{p \rightarrow p}^{\tau} \Rightarrow \overbrace{p \rightarrow 1}^{s_{p}}$ \\
\hline
\end{tabular}


Case $x=d$. Note, that $d_{2}$ is the unique represented in decomposition of $\sigma$. Then $\sigma(1)=d_{2}$. So, $\tau(d)=d_{2}$. As the result, we have the following table.

\begin{tabular}{|c|c|}
\hline$\overbrace{d \rightarrow d}^{s_{p} \cdot \pi} \Rightarrow \overbrace{d \rightarrow d_{m}}^{s_{p}}$ & $\tau \cdot \overbrace{d \rightarrow d_{2}}^{\tau} \Rightarrow \overbrace{d_{2} \rightarrow d_{2}}^{s_{p}}$ \\
\hline
\end{tabular}

Case $x=y$. Note, that the case $y=2$ is already considered. Also, $y \neq 1$ and $y \neq p$. So, $y$ is some point inside decomposition $D$. From $\pi(y)=2$ it follows that $\tau(y)=1$. As the result, we have the following table.

\begin{tabular}{|c|c|}
\hline$\overbrace{y \rightarrow y}^{s_{p}} \cdot \pi$ & $\tau \cdot s_{p}$ \\
\hline$\overbrace{p}^{s_{p}} \Rightarrow \overbrace{y \rightarrow 2}^{\pi}$ & $\overbrace{y \rightarrow 1}^{\tau} \Rightarrow \overbrace{1 \rightarrow 2}^{s_{p}}$ \\
\hline
\end{tabular}

Let $d=2$. From the construction of $\tau$ it follows that for every point $x, x \notin\{1,2, p, y\}$, we have $\pi(x) \notin\{1,2, p, y\}$. Therefore,

$$
\left(s_{p} \cdot \pi\right)(x)=\pi(x)=\tau(x)=\left(\tau \cdot s_{p}\right)(x)
$$

Hence, it is enough to show that $\left(s_{p} \cdot \pi\right)(x)=\left(\tau \cdot s_{p}\right)(x)$ for every point $x$ from $\{1,2, p, y\}$.

Case $x=1$. Note, that $d_{2}$ is the unique represented in decomposition of $\tau$. So, $\tau(1)=d_{2}$. As the result, we have the following table.

$$
\begin{array}{|c|c|}
\hline \overbrace{1 \rightarrow 2}^{s_{p} \cdot \pi} \Rightarrow \overbrace{2 \rightarrow d_{m}}^{s_{p}} & \tau \cdot s_{p} \\
\hline \overbrace{1 \rightarrow d_{2}}^{\tau} \Rightarrow \overbrace{d_{2} \rightarrow d_{2}}^{s_{p}} \\
\hline
\end{array}
$$

Case $x=2$. Note, that from $\pi(2)=d_{m}$ it follows that $\tau(2)=2$. As the result, we have the following table.

\begin{tabular}{|c|c|}
\hline$\overbrace{2 \rightarrow p}^{s_{p} \cdot \pi} \Rightarrow \overbrace{p \rightarrow p}^{s_{p}}$ & $\tau \cdot s_{p}$ \\
\hline & $\overbrace{2 \rightarrow 2}^{\tau} \Rightarrow \overbrace{2 \rightarrow p}^{s_{p}}$ \\
\hline
\end{tabular}

Case $x=p$. In this case, we obtain the following table.

\begin{tabular}{|c|c|}
\hline$\overbrace{p \rightarrow 1}^{s_{p} \cdot \pi} \Rightarrow \overbrace{1 \rightarrow 1}^{s_{p}}$ & $\tau \cdot s_{p}$ \\
\hline & $\overbrace{p \rightarrow p}^{\tau} \Rightarrow \overbrace{p \rightarrow 1}^{s_{p}}$ \\
\hline
\end{tabular}

Case $x=y$. Note, that $y \notin\{1,2, p\}$. So, $y$ is some point inside decomposition $D$. From $\pi(y)=2$ it follows that $\tau(y)=1$. As the result, we have the following table.

\begin{tabular}{|c|c|}
\hline$s_{y \rightarrow y} \cdot \pi$ & $\tau \cdot s_{p}$ \\
\hline$\overbrace{y \rightarrow y}^{s_{p}} \overbrace{y \rightarrow 2}^{\tau}$ & $\overbrace{y \rightarrow 1}^{\tau} \Rightarrow \overbrace{1 \rightarrow 2}^{s_{p}}$ \\
\hline
\end{tabular}

The proof is complete. 
Note, that the transformation of decomposition $s_{p} \cdot \pi$ to decomposition $\tau \cdot s_{p}$ do not affect any points except $p$, double $d_{2}$ (clearly $d_{2}, d_{m}$ ) and its prototype $d$. Moreover, the transformation does not change the length of decomposition.

Lemma 4. Let $\pi \in A$ be a permutation with decomposition $D=\left(d_{1}, \ldots, d_{m}\right), m \geq 3,1$ is fixed in $\pi$ over $D$ with trivial path, $\pi\left(d_{m-2}\right)=d_{m}$ and there is no $k \in \overline{1, m-3}$ such that $d_{k}=d_{m-2}$. Then for every natural number $p, p \geq 3$, there exists $\tau \in A$ such that $s_{p} \cdot \pi=\tau \cdot s_{p}$ and $\tau$ has a decomposition $\left(t_{1}, \ldots, t_{l}\right)$, where $l \leq m$ and $p \notin\left\{t_{1}, \ldots, t_{l}\right\}$.

Proof. Note, that if $p \notin\left\{d_{1}, \ldots, d_{m}\right\}$, then the statement holds by Lemma 3.

Let $p \in\left\{d_{1}, \ldots, d_{m}\right\}$. Consider seven different cases:

1) $d_{2}=p$;

2) $d_{2} \neq p, d_{m-1}, d_{m-2} \neq p$;

3) $d_{2} \neq p, d_{m-1}=p$ and $d_{m-2} \neq p$;

4) $d_{2} \neq p, d_{m-1} \neq p, d_{m-2}=p$ and $\pi^{-1}(p) \neq 2$;

5) $d_{2} \neq p, d_{m-1}, d_{m-2}=p$ and $d:=\pi^{-1}(p) \neq 2$;

6) $d_{2} \neq p, d_{m-1} \neq p, d_{m-2}=p$ and $\pi^{-1}(p)=2$;

7) $d_{2} \neq p, d_{m-1}, d_{m-2}=p$ and $\pi^{-1}(p)=2$.

1) Let $d_{2}=p$. From Proposition 1 it follows that:

(a) if $d_{1} \neq p$, then

$$
\begin{aligned}
s_{p} \cdot \pi & =[p]_{S} \cdot\left[d_{1}, p, d_{3}, \ldots, d_{m-1}, p\right]_{S}=\left[p, d_{1}, p, d_{3}, \ldots, d_{m-1}, p\right]_{S} \\
& =\left[d_{1}, d_{1}, p, p, p, d_{3}, \ldots, d_{m-1}, p\right]_{S}=\left[d_{1}, d_{1}, d_{3}, \ldots, d_{m-1}, p\right]_{S},
\end{aligned}
$$

so, $\tau=\left[d_{1}, d_{1}, d_{3}, \ldots, d_{m-1}\right]_{S}$ with the length equals to $m-1$;

(b) if $d_{1}=p$, then

$s_{p} \cdot \pi=[p]_{S} \cdot\left[p, p, d_{3}, \ldots, d_{m-1}, p\right]_{S}=\left[p, p, p, d_{3}, \ldots, d_{m-1}, p\right]_{S}=\left[d_{3}, \ldots, d_{m-1}\right]_{S} \cdot[p]_{S}$, so, $\tau=\left[d_{3}, \ldots, d_{m-1}\right]_{S}$ with the length equals to $m-3$.

2) Let $d_{2} \neq p, d_{m-1}, d_{m-2} \neq p$. Construct $\tau$ as follows

$$
\tau=\left[d_{m-2}, t_{1}, \ldots, t_{m-1}\right]_{s}, \quad \text { where } t_{i}= \begin{cases}d_{m-2}, & \text { if } d_{i}=p \\ d_{i}, & \text { otherwise }\end{cases}
$$

Let $\sigma=\left[d, t_{1}, \ldots, t_{m-3}\right]_{S}$. Note, that $\tau=\sigma \cdot\left[t_{m-2}, t_{m-1}\right]_{S}=\sigma \cdot\left[d_{m-2}, d_{m-1}\right]_{S}$.

From the construction of $\tau$ it follows that for every point $x, x \notin\left\{1,2, d_{m-2}, p, \pi^{-1}(p)\right\}$, we have $\pi(x) \notin\left\{1,2, d_{m-2}, p, \pi^{-1}(p)\right\}$. Therefore, $\left(s_{p} \cdot \pi\right)(x)=\pi(x)=\tau(x)=\left(\tau \cdot s_{p}\right)(x)$.

Hence, it is enough to show that $\left(s_{p} \cdot \pi\right)(x)=\left(\tau \cdot s_{p}\right)(x)$ for every point $x$ from $\left\{1,2, d_{m-2}, p, \pi^{-1}(p)\right\}$. 
Case $x=1$. Note, that

(a) if $\pi(2)=2$, then the path of 2 in $\pi$ over $D$ ends on the $m$ th position. So, $\sigma(2)=1$, from which it follows that $\tau(1)=1$;

(b) if $\pi(2)=p$, then $\sigma(1)=d_{m-2}$. Then we have $\overbrace{1 \rightarrow d_{m-2}}^{\sigma} \Rightarrow \overbrace{d_{m-2} \rightarrow 1}^{\left(1,2, d_{m-2}\right)} \Rightarrow \overbrace{1 \rightarrow 2}^{\left(1,2, d_{m-1}\right)}$, which means that $\tau(1)=2$;

(c) if $\pi(2) \neq 2, p$, then $\pi(2)=\tau(1)$.

As the result, we have the following table.

\begin{tabular}{|c|c|c|}
\hline & $s_{p} \cdot \pi$ & $\tau \cdot s_{p}$ \\
\hline & 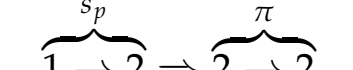 & $\overbrace{1 \rightarrow 1}^{\tau} \rightarrow \overbrace{1 \rightarrow 2}^{s_{p}}$ \\
\hline (-) & & \\
\hline$\pi(2)=p$ & $\underset{s_{p}}{1} \rightarrow 2 \Rightarrow \underset{\pi}{2} \underset{\pi}{\rightarrow} p$ & $\tau_{\tau} 1 \rightarrow 2 \Rightarrow 2 \rightarrow s_{s_{p}}^{p}$ \\
\hline$\pi(2) \neq 2, p$ & $\overparen{1 \rightarrow 2} \Rightarrow 2 \rightarrow \pi(2)$ & $\overparen{1 \rightarrow \pi(2)} \Rightarrow \pi(2) \rightarrow \pi(2)$ \\
\hline
\end{tabular}

Case $x=2$. Note, that

(a) if $\pi(p) \neq p$, then $\tau(2)=\pi(p)$, based on construction $\tau$ from $\pi$;

(b) if $\pi(p)=p$, then $\sigma(2)=d_{m-2}$. So $\overbrace{2 \rightarrow d_{m-2}}^{\sigma} \Rightarrow \overbrace{d_{m-2} \rightarrow 1}^{\left(1,2, d_{m-2}\right)} \Rightarrow \overbrace{1 \rightarrow 2}^{\left(1,2, d_{m-1}\right)}$, which means that $\tau(2)=2$.

As the result, we have the following table.

\begin{tabular}{|l|c|c|}
\hline$\pi(p) \neq p$ & $\overbrace{2 \rightarrow p}^{s_{p}} \Rightarrow \overbrace{p \rightarrow \pi(p)}^{s_{p} \cdot \pi}$ & $\tau \cdot s_{2 \rightarrow \pi(p)}^{\tau} \Rightarrow \overbrace{\pi(p) \rightarrow \pi(p)}^{\tau}$ \\
$\pi(p)=p$ & $\overbrace{2 \rightarrow p}^{s_{p}} \Rightarrow \overbrace{p \rightarrow p}^{s_{p}}$ & $\overbrace{2 \rightarrow p}^{\tau} \Rightarrow \overbrace{p \rightarrow 2}^{s_{p}}$ \\
\hline
\end{tabular}

Case $x=d_{m-2}$. Note, that $\pi\left(d_{m-2}\right)=d_{m}=d_{2}=\tau\left(d_{m-2}\right)$. As the result, we have the following table.

\begin{tabular}{|c|c|}
\hline$\overbrace{d_{m-2} \rightarrow d_{m-2}}^{s_{p} \cdot \pi} \Rightarrow \overbrace{d_{m-2} \rightarrow d_{m}}^{s_{p}}$ & $\tau \cdot \overbrace{d_{m-2} \rightarrow d_{2}}^{\tau} \Rightarrow \overbrace{d_{2} \rightarrow d_{2}}^{s_{p}}$ \\
\hline
\end{tabular}

Case $x=p$. In this case, we have the following table.

\begin{tabular}{|c|c|}
\hline$\overbrace{p \rightarrow 1}^{s_{p} \cdot \pi} \Rightarrow \overbrace{1 \rightarrow 1}^{s_{p}}$ & $\tau \cdot s_{p}$ \\
\hline & $\overbrace{p \rightarrow p}^{\tau} \Rightarrow \overbrace{p \rightarrow 1}^{s_{p}}$ \\
\hline
\end{tabular}


Case $x=\pi^{-1}(p)$. Let $d=\pi^{-1}(p)$. Note, that cases $d=2$ or $d=p$ are already considered.

Also, $d$ doesn't equal 1. Then for $d$ in $\tau$ we have $\overbrace{d \rightarrow d_{m-2}}^{\sigma} \Rightarrow \overbrace{d_{m-2} \rightarrow 1}^{\left(1,2, d_{m-2}\right)} \Rightarrow \overbrace{1 \rightarrow 2}^{\left(1,2, d_{m-1}\right)}$. As the result, we have the following table.

\begin{tabular}{|c|c|c|}
\hline & $s_{p} \cdot \pi$ & $\tau \cdot s_{p}$ \\
\hline$d=\pi^{-1}(p), d \neq 2, p$ & $\overbrace{d \rightarrow d}^{s_{p}} \Rightarrow \overbrace{d \rightarrow p}^{\pi}$ & $\overbrace{d \rightarrow 2}^{\tau} \Rightarrow \overbrace{2 \rightarrow p}^{s_{p}}$ \\
\hline
\end{tabular}

3) Let $d_{2} \neq p, d_{m-1}=p$ and $d_{m-2} \neq p$. Construct $\tau$ as follows

$$
\tau=\left[d_{m-2}, t_{1}, \ldots, t_{m-2}\right]_{s}, \quad \text { where } t_{i}= \begin{cases}d_{m-2}, & \text { if } d_{i}=p, \\ d_{i}, & \text { otherwise. }\end{cases}
$$

Let $\sigma=\left[d_{m-2}, t_{1}, \ldots, t_{m-3}\right]_{S}$. Note, that $\tau=\sigma \cdot\left[t_{m-2}\right]_{S}=\sigma \cdot\left[d_{m-2}\right]_{s}$.

From the construction of $\tau$ it follows that for every point $x, x \notin\left\{1,2, d_{m-2}, p\right\}$, we have $\pi(x) \notin\left\{1,2, d_{m-2}, p\right\}$. Therefore, $\left(s_{p} \cdot \pi\right)(x)=\pi(x)=\tau(x)=\left(\tau \cdot s_{p}\right)(x)$. Hence, it is enough to show that $\left(s_{p} \cdot \pi\right)(x)=\left(\tau \cdot s_{p}\right)(x)$ for every point $x$ from $\left\{1,2, d_{m-2}, p\right\}$.

Case $x=1$. Note, that

(a) if $\pi(2)=2$, then in the decomposition of $\tau: 1 \stackrel{1}{\rightarrow} 2 \stackrel{2}{\rightarrow} t_{2} \Rightarrow \ldots \stackrel{x}{\rightarrow} d_{m-2} \stackrel{x, m-2}{\longrightarrow}$ $d_{m-2} \stackrel{m-2}{\longrightarrow} 1$, where $x$ is the last position of $p$, before $m-1$. So, $\tau(2)=1$;

(b) if $\pi(2)=p$, then the last point of 2 was the $(m-1)$ th position in the decomposition $D$. As the result, $\sigma(1)=1$. So, $\overbrace{1 \rightarrow 1}^{\sigma} \Rightarrow \overbrace{1 \rightarrow 2}^{\left(1,2, d_{2}\right)}$, which means that $\tau(1)=2$;

(c) if $\pi(2) \neq p$, then $\sigma(1)=\pi(2) \neq d_{m-2}$. So, $\overbrace{1 \rightarrow \pi(2)}^{\sigma} \Rightarrow \overbrace{\pi(2) \rightarrow \pi(2)}^{\left(1,2, d_{m-2}\right)}$, which means that $\tau(1)=\pi(2)$.

As the result, we have the following table.

\begin{tabular}{|c|c|c|}
\hline & $s_{p} \cdot \pi$ & $\tau \cdot s_{p}$ \\
\hline$\pi(2)=2$ & $\overbrace{1 \rightarrow 2}^{s p} \Rightarrow \overbrace{2 \rightarrow 2}^{\pi}$ & $\overbrace{1 \rightarrow 1}^{\tau} \Rightarrow \overbrace{1 \rightarrow 2}^{s_{p}}$ \\
\hline$\pi(2)=p$ & $\overbrace{1 \rightarrow 2}^{\rightarrow} \Rightarrow \overbrace{2 \rightarrow p}$ & $\overbrace{1 \rightarrow 2} \Rightarrow \overbrace{2 \rightarrow p}$ \\
\hline$\pi(2) \neq 2, p$ & $\overbrace{1 \rightarrow 2} \Rightarrow \overbrace{2 \rightarrow \pi(2)}$ & $\overbrace{1 \rightarrow \pi(2)} \Rightarrow \overbrace{\pi(2) \rightarrow \pi(2)}$ \\
\hline
\end{tabular}

Case $x=2$. Note, that

(a) if $\pi(p) \neq p$, then $\sigma(2)=\pi(p)$, because of $2 \stackrel{1}{\rightarrow} d_{m-2}$ in $\tau$. As the result, we have that $\overbrace{2 \rightarrow \pi(p)}^{\sigma} \Rightarrow \overbrace{\pi(p) \rightarrow \pi(p)}^{\left(1,2, d_{m-2}\right)}$, which means that $\tau(2)=\pi(p)$;

(b) if $\pi(p)=p$, then the last point in the path of action of 2 over the decomposition $D$ will be the $(m-1)$ th position. As the result, $\sigma(2)=1$. So, $\overbrace{2 \rightarrow 1}^{\sigma} \Rightarrow \overbrace{1 \rightarrow 2}^{\left(1,2, d_{m-2}\right)}$, which means that $\tau(2)=2$. 
As the result, we have the following table.

\begin{tabular}{|l|l|c|}
\hline$\pi(p) \neq p$ & $\overbrace{2 \rightarrow p}^{s_{p}} \Rightarrow \overbrace{p \rightarrow \pi(p)}^{s_{p} \cdot \pi}$ & $\overbrace{2 \rightarrow \pi(p)}^{\tau} \Rightarrow \overbrace{\pi(p) \rightarrow \pi(p)}^{\tau}$ \\
$\pi(p)=p$ & $\overbrace{2 \rightarrow p}^{s_{p}} \Rightarrow \overbrace{p \rightarrow p}^{s_{p}}$ & $\overbrace{2 \rightarrow 2}^{\tau} \Rightarrow \overbrace{2 \rightarrow p}^{s_{p}}$ \\
\hline
\end{tabular}

Case $x=d_{m-2}$. Note, that $\tau\left(d_{m-2}\right)=d_{2}$. As the result, we have the following table.

\begin{tabular}{|c|c|}
\hline$\overbrace{d_{m-2} \rightarrow d_{m-2}}^{s_{p} \cdot \pi} \Rightarrow \overbrace{d_{m-2} \rightarrow d_{m}}^{s_{p}}$ & \multicolumn{1}{c|}{$\tau \cdot \overbrace{d_{m-2} \rightarrow d_{2}}^{\tau} \Rightarrow \overbrace{d_{2} \rightarrow d_{2}}^{s_{p}}$} \\
\hline
\end{tabular}

Case $x=p$. Note, that $\tau(p)=p$, because there are no point $p$ in the decomposition of $\tau$. As the result, we have the following table.

\begin{tabular}{|c|c|}
\hline$\overbrace{p \rightarrow 1}^{s_{p} \cdot \pi} \Rightarrow \overbrace{1 \rightarrow 1}^{s_{p}}$ & $\tau \cdot s_{p}$ \\
\hline & $\overbrace{p \rightarrow p}^{\tau} \Rightarrow \overbrace{p \rightarrow 1}^{s_{p}}$ \\
\hline
\end{tabular}

Case $x=\pi^{-1}(p)$. Let $d=\pi^{-1}(p)$. Note, that cases $d=2$ or $d=p$ are already considered. Also, $d$ does not equal to 1 . Then for $d$ in $\tau$ we have $\overbrace{d \rightarrow 1}^{\sigma} \Rightarrow \overbrace{1 \rightarrow 2}^{\left(1,2, d_{m-2}\right)}$, which means that $\tau(d)=2$. As the result, we have the following table.

\begin{tabular}{|l|l|l|}
\hline & $s_{p} \cdot \pi$ & $\tau \cdot s_{p}$ \\
\hline$d=\pi^{-1}(p), d \neq 2, p$ & $\overbrace{d \rightarrow d}^{s_{p}} \Rightarrow \overbrace{d \rightarrow p}^{\pi}$ & $\overbrace{d \rightarrow 2}^{\tau} \Rightarrow \overbrace{2 \rightarrow p}^{s_{p}}$ \\
\hline
\end{tabular}

Note, that in this case, the length of $\tau$ over $S$ equals to $m-1$.

4) Let $d_{2} \neq p, d_{m-1} \neq p, d_{m-2}=p$ and $\pi^{-1}(p) \neq 2$. Let $d=\pi^{-1}(p)$. Note, that in this case $d_{m-2}$ is unique representation of point $p$ in $D$. Construct $\tau$ as follows

$$
\tau=\left[d, d_{1}, d_{2}, \ldots, d_{m-3}, d_{2}, d_{m-1}\right]_{S},
$$

where $d=\pi^{-1}(p)$.

From the construction of $\tau$ it follows that for every point $x, x \notin\left\{1,2, d_{m-2}=p, d\right\}$ we have $\pi(x) \notin\left\{1,2, d_{m-2}=p, d\right\}$. Therefore, $\left(s_{p} \cdot \pi\right)(x)=\pi(x)=\tau(x)=\left(\tau \cdot s_{p}\right)(x)$. Hence, it is enough to show that $\left(s_{p} \cdot \pi\right)(x)=\left(\tau \cdot s_{p}\right)(x)$ for every point $x$ from $\left\{1,2, d_{m-2}=p, d\right\}$.

Case $x=1$. Note, that

(a) if $\pi(2)=2$, then $\tau(1)=1$;

(b) if $\pi(2) \neq 2$, then $\tau(1)=\pi(2)$.

As the result, we have the following table.

\begin{tabular}{|l|l|l|}
\hline$\pi(2)=2$ & $\overbrace{1 \rightarrow 2}^{s_{p}} \Rightarrow \overbrace{2 \rightarrow 2}^{s_{p} \cdot \pi}$ & $\overbrace{1 \rightarrow 1}^{\tau} \Rightarrow \overbrace{1 \rightarrow 2}^{s_{1 \rightarrow 2}}$ \\
$\pi(2) \neq 2$ & $\overbrace{1 \rightarrow 2}^{s_{p}} \Rightarrow \overbrace{2 \rightarrow \pi(2)}^{s_{p}}$ & $\overbrace{1 \rightarrow \pi(2)}^{\tau} \Rightarrow \overbrace{\pi(2) \rightarrow \pi(2)}^{s_{p}}$ \\
\hline
\end{tabular}


Case $x=2$. Note, that $\tau(2)=\left[d_{1}, \ldots, d_{m-3}, d_{2}, d_{m-2}\right]_{S}(d)=d_{2}$. As the result, we have the following table.

$$
\begin{array}{|c|c|}
\hline \overbrace{2 \rightarrow p}^{s_{p}} \Rightarrow \overbrace{p \rightarrow d_{m}}^{s_{p}} \pi & \overbrace{2 \rightarrow d_{2}}^{\tau} \Rightarrow \overbrace{d_{2} \rightarrow d_{2}}^{s_{p}} \\
\hline
\end{array}
$$

Case $x=p$. In this case we have $\tau(p)=p$. As the result, we obtain the following table.

\begin{tabular}{|c|c|}
\hline$\overbrace{p \rightarrow 1}^{s_{p} \cdot \pi} \Rightarrow \overbrace{1 \rightarrow 1}^{s_{p}}$ & $\tau \cdot s_{p}$ \\
\hline & $\overbrace{p \rightarrow p}^{\tau} \Rightarrow \overbrace{p \rightarrow 1}^{s_{p}}$ \\
\hline
\end{tabular}

Case $x=d$. Note, that the case $d=p$ is already considered. So, in the decomposition of $\tau$, we have the path $d \stackrel{1}{\rightarrow} 1 \stackrel{2}{\rightarrow} 2 \stackrel{3}{\rightarrow} d_{2} \stackrel{3, m-1}{\longrightarrow} d_{2} \stackrel{m-1}{\rightarrow} 1 \stackrel{m}{\rightarrow} 2$. So, $\tau(d)=2$. As the result, we have the following table.

\begin{tabular}{|c|c|}
\hline$\overbrace{d \rightarrow d}^{s_{p} \cdot \pi} \Rightarrow \overbrace{d \rightarrow p}^{s_{p}}$ & $\tau \cdot s_{p}$ \\
\hline & $\overbrace{d \rightarrow 2}^{\tau} \Rightarrow \overbrace{2 \rightarrow p}^{s_{p}}$ \\
\hline
\end{tabular}

5) Let $d_{2} \neq p, d_{m-1}=d_{m-2}=p$ and $\pi^{-1}(p) \neq 2$. Let $d=\pi^{-1}(p)$. Note, that in this case, $d_{m-2}, d_{m-1}$ are unique represented of the point $p$ in $D$. Construct $\tau$ as follows

$$
\tau=\left[d, d_{1}, d_{2}, \ldots, d_{m-3}, d_{2}, d_{2}\right]_{S} .
$$

From the construction of $\tau$ it follows that for every point $x, x \notin\left\{1,2, d_{m-2}=p, d\right\}$, we have $\pi(x) \notin\left\{1,2, d_{m-2}=p, d\right\}$. Therefore, $\left(s_{p} \cdot \pi\right)(x)=\pi(x)=\tau(x)=\left(\tau \cdot s_{p}\right)(x)$. Hence, it is enough to show that $\left(s_{p} \cdot \pi\right)(x)=\left(\tau \cdot s_{p}\right)(x)$ for every point $x$ from $\left\{1,2, d_{m-2}=p, d\right\}$.

Case $x=1$. Note, that
(a) if $\pi(2)=2$, then $\tau(1)=1$;
(b) if $\pi(2) \neq 2$, then $\tau(1)=\pi(2)$.

As the result, we have the following table.

\begin{tabular}{|l|l|l|}
\hline & $\overbrace{}^{s_{p} \cdot \pi}$ & \multicolumn{1}{|c|}{$\tau \cdot s_{p}$} \\
\hline$\pi(2)=2$ & $\overbrace{1 \rightarrow 2}^{s_{p}} \Rightarrow \overbrace{2 \rightarrow 2}^{\pi}$ & $\overbrace{1 \rightarrow 1}^{\tau} \Rightarrow \overbrace{1 \rightarrow 2}^{s_{p}}$ \\
$\overbrace{1 \rightarrow 2}^{s_{p}} \Rightarrow \overbrace{2 \rightarrow \pi(2)}^{\pi}$ & $\overbrace{1 \rightarrow \pi(2)}^{\tau} \Rightarrow \overbrace{\pi(2) \rightarrow \pi(2)}^{s_{p}}$ \\
\hline
\end{tabular}

Case $x=2$. Note, that $\tau(2)=\left[d_{1}, \ldots, d_{m-3}, d_{2}, d_{2}\right]_{S}(d)=d_{2}$. As the result, we have the following table.

\begin{tabular}{|c|c|}
\hline$\overbrace{2 \rightarrow p}^{s_{p}} \Rightarrow \overbrace{p \rightarrow d_{2}}^{s_{p} \cdot \pi}$ & $\tau \cdot \overbrace{2 \rightarrow d_{2}}^{\tau} \Rightarrow \overbrace{d_{2} \rightarrow d_{2}}^{s_{p}}$ \\
\hline
\end{tabular}


Case $x=p$. In this case $\tau(p)=p$. As the result, we have the following table.

\begin{tabular}{|c|c|}
\hline$\overbrace{p \rightarrow 1}^{s_{p} \cdot \pi} \Rightarrow \overbrace{1 \rightarrow 1}^{s_{p}}$ & $\tau \cdot s_{p}$ \\
\hline$\overbrace{p \rightarrow p}^{\tau} \Rightarrow \overbrace{p \rightarrow 1}^{s_{p}}$ \\
\hline
\end{tabular}

Case $x=d$. Note, that the case $d=p$ is already considered. So, in the decomposition of $\tau$, we have the path $d \stackrel{1}{\rightarrow} 1 \stackrel{2}{\rightarrow} 2 \stackrel{3}{\rightarrow} d_{2} \stackrel{3, m-1}{\longrightarrow} d_{2} \stackrel{m-1}{\rightarrow} 1 \stackrel{m}{\rightarrow} 2$. So, $\tau(d)=2$. As the result, we have the following table.

\begin{tabular}{|c|c|}
\hline$\overbrace{d \rightarrow d}^{s_{p} \cdot \pi} \Rightarrow \overbrace{d \rightarrow p}^{s_{p}}$ & $\tau \cdot s_{p}$ \\
\hline$\overbrace{d \rightarrow 2}^{\tau} \Rightarrow \overbrace{2 \rightarrow p}^{s_{p}}$ \\
\hline
\end{tabular}

6) Let $d_{2} \neq p, d_{m-1} \neq p, d_{m-2}=p$ and $\pi^{-1}(p)=2$. Note, that in this case $d_{m-2}$ is the unique representation of the point $p$ in $D$. Construct $\tau$ as follows

$$
\tau=\left[d_{1}, d_{2}, \ldots, d_{m-3}, d_{2}, d_{m-1}\right]_{S} .
$$

From the construction of $\tau$ it follows that for every point $x, x \notin\left\{1,2, d_{m-2}=p\right\}$, we have $\pi(x) \notin\{1,2, p\}$. Therefore, $\left(s_{p} \cdot \pi\right)(x)=\pi(x)=\tau(x)=\left(\tau \cdot s_{p}\right)(x)$. Hence, it is enough to show that $\left(s_{p} \cdot \pi\right)(x)=\left(\tau \cdot s_{p}\right)(x)$ for every point $x$ from $\{1,2, p\}$.

Case $x=1$. Note, that in decomposition of $\tau$ there is the next path of the point 1 , namely $1 \stackrel{1}{\rightarrow} 2 \stackrel{2}{\rightarrow} d_{2} \stackrel{2, m-2}{\longrightarrow} d_{2} \stackrel{m-2}{\longrightarrow} 1 \stackrel{m-1}{\longrightarrow} 2$. So, $\tau(1)=2$. As the result, we have the following table.

\begin{tabular}{|c|c|}
\hline$\overbrace{1 \rightarrow 2}^{s_{p} \cdot \pi} \Rightarrow \overbrace{2 \rightarrow p}^{s_{p}}$ & $\tau \cdot s_{p}$ \\
\hline$\overbrace{1 \rightarrow 2}^{\tau} \Rightarrow \overbrace{2 \rightarrow p}^{s_{p}}$ \\
\hline
\end{tabular}

Case $x=2$. Based on the fact that $d_{m-2}$ is the unique representation of $p$ in $D$, we have that $\pi(p)=d_{m}$. In the same time, $\pi(2)=p=d_{m-2}$, which means that $\tau(2)=d_{2}$. As the result, we have the following table.

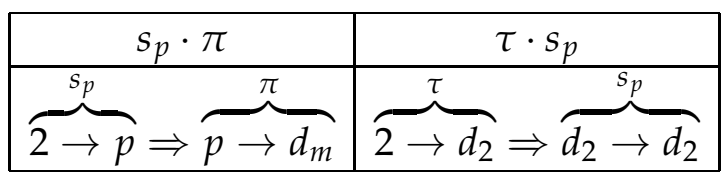

Case $x=p$. Note, that $\tau(p)=p$. As the result, we have the following table.

\begin{tabular}{|c|c|}
\hline$\overbrace{p \rightarrow 1}^{s_{p} \cdot \pi} \Rightarrow \overbrace{1 \rightarrow 1}^{s_{p}}$ & $\tau \cdot s_{p}$ \\
\hline & $\overbrace{p \rightarrow p}^{\tau} \Rightarrow \overbrace{p \rightarrow 1}^{s_{p}}$ \\
\hline
\end{tabular}

7) Let $d_{2} \neq p, d_{m-1}=d_{m-2}=p$ and $\pi^{-1}(p)=2$. Note that in this case, $d_{m-2}, d_{m-1}$ are the unique representations of $p$ in $D$. Construct $\tau$ as follows

$$
\tau=\left[d_{1}, d_{2}, \ldots, d_{m-3}, d_{2}, d_{2}\right]_{S} .
$$

From the construction of $\tau$ it follows that for every point $x, x \notin\left\{1,2, d_{m-2}=p\right\}$, we have $\pi(x) \notin\{1,2, p\}$. Therefore, $\left(s_{p} \cdot \pi\right)(x)=\pi(x)=\tau(x)=\left(\tau \cdot s_{p}\right)(x)$. Hence, it is enough to show that $\left(s_{p} \cdot \pi\right)(x)=\left(\tau \cdot s_{p}\right)(x)$ for every point $x$ from $\{1,2, p\}$. 
Case $x=1$. Note, that there is the next path of point 1 in the decomposition of $\tau$, namely $1 \stackrel{1}{\rightarrow} 2 \stackrel{2}{\rightarrow} d_{2} \stackrel{2, m-2}{\longrightarrow} d_{2} \stackrel{m-2}{\longrightarrow} 1 \stackrel{m-1}{\longrightarrow} 2$. So, $\tau(1)=2$. As the result, we have the following table.

\begin{tabular}{|c|c|}
\hline$\overbrace{1 \rightarrow 2}^{s_{p} \cdot \pi} \Rightarrow \overbrace{2 \rightarrow p}^{s_{p}}$ & $\tau \cdot s_{p}$ \\
\hline & $\overbrace{1 \rightarrow 2}^{\tau} \Rightarrow \overbrace{2 \rightarrow p}^{s_{p}}$ \\
\hline
\end{tabular}

Case $x=2$. Based on the fact that $d_{m-2}$ is the unique representation of $p$ in $D$, we have that $\pi(p)=d_{m}$. In the same time, $\pi(2)=p=d_{m-1}$, which means that $\tau(2)=d_{2}$. As the result, we have the following table.

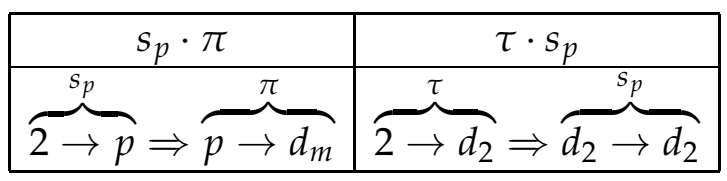

Case $x=p$. Note, that $\tau(p)=p$. As the result, we have the following table.

\begin{tabular}{|c|c|}
\hline$\overbrace{p \rightarrow 1}^{s_{p} \cdot \pi} \Rightarrow \overbrace{1 \rightarrow 1}^{s_{p}}$ & $\tau \cdot s_{p}$ \\
\hline & $\overbrace{p \rightarrow p}^{\tau} \Rightarrow \overbrace{p \rightarrow 1}^{s_{p}}$ \\
\hline
\end{tabular}

The proof of lemma is completed.

Lemma 5. Let $\pi$ be a permutation with decomposition $D=\left(d_{1}, d_{2}, \ldots, d_{m}\right), m \geq 3,1$ is fixed in $\pi$ over $D$ with trivial path and $\pi^{-1}\left(d_{2}\right) \neq 2$. Then for every natural number $p, p \geq 3$, there exists $\tau \in A$ such that $s_{p} \cdot \pi=\tau \cdot s_{p}$ and $\tau$ has a decomposition $\left(t_{1}, \ldots, t_{l}\right)$, where $l \leq m$ and $p \notin\left\{t_{1}, \ldots, t_{l}\right\}$.

Proof. Note, that the case, when there is no such $k \in \overline{1, m}$ that $d_{k}=p$, is already proved in Lemma 3, and the case, when $\pi^{-1}\left(d_{m}\right)=d_{m-2}$ with no such $k \in \overline{1, m-3}$ that $d_{k}=d_{m-2}$, is already proved in Lemma 4.

The basis. Let $d=\pi^{-1}\left(d_{m}\right)$. Note, that due to assumptions of the lemma, the decomposition of the permutation $\pi$ requires: 2 positions for $d_{2}, d_{m}$; at least 2 positions for non-trivial path of the point $d_{m} ; 1$ position for $d_{m-1}$. The last means that $m \geq 5$.

We will prove the basis for cases $m=5,6$.

1) If $m=5$, then $d=2$ and there exists the following decomposition of $\pi$, namely $\pi=\left[d_{1}, d_{2}, d_{3}, d_{4}, d_{5}\right]_{S}$, where $d_{1}=d_{3}, d_{2}=d_{4}, d_{5} \neq d_{2}$ and $d_{5} \neq d_{4}$. Based on Lemma 3 , it is enough to consider three cases depending on $p$ from $\left\{d_{1}, d_{2}, d_{5}\right\}$. In case $p=d_{1}$ let $\tau=\left[d_{2}, d_{2}, d_{4}\right]_{S}$. It follows that $s_{d_{1}} \cdot \pi=\tau \cdot s_{d_{1}}$. In case $p=d_{2}$ let $\tau=\left[d_{4}\right]_{S}$. It follows that $s_{d_{2}} \cdot \pi=\tau \cdot s_{d_{2}}$. In case $p=d_{5}$ let $\tau=\left[d_{2}, d_{2}\right]_{S}$. It follows that $s_{d_{4}} \cdot \pi=\tau \cdot s_{d_{4}}$. In each case we used direct calculations.

2) If $m=6$, then $d$ can be equal to 2 , or not. We consider the next three cases: $d=2$ and $d_{3} \neq d_{5} ; d=2$ and $d_{3}=d_{5} ; d \neq 2$.

Let $d=2$. Then we have the next decomposition of $\pi$, namely $\pi=\left[d_{1}, d_{2}, d_{3}, d_{4}, d_{5}, d_{6}\right]_{S}$, where $d_{1}=d_{4}, d_{2}=d_{6}$ and $d_{3}, d_{5}$ are different from $d_{1}, d_{2}$.

Let $d_{3} \neq d_{5}$. Based on Lemma 3 it is enough to consider four cases depending on $p$ from $\left\{d_{1}, d_{2}, d_{3}, d_{5}\right\}$. In case $p=d_{1}$ let $\tau=\left[d_{3}, d_{2}, d_{5}\right]_{S}$. It follows that $s_{d_{1}} \cdot \pi=\tau \cdot s_{d_{1}}$. In case $p=d_{2}$ let $\tau=\left[d_{1}, d_{3}, d_{3}, d_{5}\right]_{S}$. It follows that $s_{d_{2}} \cdot \pi=\tau \cdot s_{d_{2}}$. In case $p=d_{3}$ let $\tau=\left[d_{5}, d_{2}, d_{1}, d_{1}\right]_{s}$. It 
follows that $s_{d_{3}} \cdot \pi=\tau \cdot s_{d_{3}}$. In case $p=d_{5}$ let $\tau=\left[d_{1}, d_{2}, d_{3}, d_{1}\right]_{S}$. It follows that $s_{d_{5}} \cdot \pi=\tau \cdot s_{d_{5}}$. In each case we used direct calculations.

Let $d_{3}=d_{5}$. Note, that $\pi\left(d_{3}\right)=d_{3}$. Based on Lemma 3 it is enough to consider two cases depending on $p$ from $\left\{d_{1}, d_{2}\right\}$. In case $p=d_{1}$ let $\tau=\left[d_{1}, d_{2}, d_{1}\right]_{s}$. It follows that $s_{d_{1}} \cdot \pi=\tau \cdot s_{d_{1}}$. In case $p=d_{2}$ let $\tau=\left[d_{1}\right]_{s}$. It follows that $s_{d_{2}} \cdot \pi=\tau \cdot s_{d_{2}}$. In each case we used direct calculations.

Let $d \neq 2$. Then there is the next decomposition of $\pi$, namely $\pi=\left[d_{1}, d_{2}, d_{3}, d_{4}, d_{5}, d_{6}\right]_{s}$, where $d_{3}=d_{4}, d_{2}=d_{6}$ and points $d_{1}, d_{2}, d_{3}, d_{5}$ are pairwise different. From Proposition 1 we have $\pi=\left[d_{1}, d_{2}, d_{3}, d_{3}, d_{5}, d_{2}\right]_{s}=\left[d_{2}, d_{2}, d_{1}, d_{1}, d_{3}, d_{3}, d_{5}, d_{2}\right]_{s}=\left[d_{2}, d_{2}, d_{3}, d_{1}, d_{5}, d_{2}\right]_{s}$. Statement is directly implied from Lemma 4.

Induction step: case $m$ under assumption that for $l<m$ the statement holds. Let $d=\pi^{-1}\left(d_{m}\right)$. Consider cases of $d$.

Let $d=2$. Consider different cases.

1) Let $d_{2}=d_{m}=p$. There are possible the next two options. If $d_{1}=p$, then by Proposition 1 we have $s_{p} \cdot \pi=[p]_{S} \cdot\left[p, p, d_{3}, \ldots, d_{m-1}, p\right]_{S}=\left[d_{3}, \ldots, d_{m-1}\right]_{S} \cdot[p]_{S}=\tau \cdot s_{p}$. If $d_{1} \neq p$, then based on Proposition 1 we obtain

$$
\begin{aligned}
s_{p} \cdot \pi=[p]_{S} \cdot\left[d_{1}, p, d_{3}, \ldots, d_{m-1}, p\right]_{S} & =\left[d_{1}, d_{1}, p, p, p, d_{3}, \ldots, d_{m-1}, p\right]_{S} \\
& =\left[d_{1}, d_{1}, d_{3}, \ldots, d_{m-1}\right]_{S} \cdot[p]_{S}=\tau \cdot s_{p}
\end{aligned}
$$

2) Let $d_{2} \neq p, d_{m} \neq p$. Denote $\pi(p)$ by $d$. There are possible the following three options.

i) Let $d=2, d_{m-1}=p$ and there is no $t \in \overline{1, m-2}$ such that $p=d_{t}$. Construct $\tau$ as follows $\tau=\left[d_{1}, d_{2}, \ldots, d_{m-2}\right]_{S}$.

From the construction of $\tau$ it follows that for every point $x, x \notin\left\{1,2, p, p^{-1}\right\}$, we have $\pi(x) \notin\left\{1,2, p, p^{-1}\right\}$. Therefore, $\left(s_{p} \cdot \pi\right)(x)=\pi(x)=\tau(x)=\left(\tau \cdot s_{p}\right)(x)$. Hence, it is enough to show that $\left(s_{p} \cdot \pi\right)(x)=\left(\tau \cdot s_{p}\right)(x)$ for every point $x$ from $\left\{1,2, p, p^{-1}\right\}$.

Case $x=1$. Note, that $d_{2}$ is the unique point in the decomposition of $\tau$. So, $\tau(1)=d_{2}$. As the result, we have the following table.

\begin{tabular}{|c|c|}
\hline$\overbrace{1 \rightarrow 2}^{s_{p} \cdot \pi} \Rightarrow \overbrace{2 \rightarrow d_{m}}^{s_{p}}$ & $\overbrace{1 \rightarrow d_{2}}^{\tau} \Rightarrow \overbrace{d_{2} \rightarrow d_{2}}^{s_{p}}$ \\
\hline
\end{tabular}

Case $x=2$. From $\pi(2)=d_{m}$ it follows that $\tau(2)=1$. As the result, we have the following table.

\begin{tabular}{|c|c|}
\hline$\overbrace{2 \rightarrow p}^{s_{p} \cdot \pi} \Rightarrow \overbrace{p \rightarrow 2}^{s_{p}}$ & $\pi \cdot s_{p}$ \\
\hline & $\overbrace{2 \rightarrow 1}^{\tau} \Rightarrow \overbrace{1 \rightarrow 2}^{s_{p}}$ \\
\hline
\end{tabular}

Case $x=p$. In this case, we have the following table.

\begin{tabular}{|c|c|}
\hline$\overbrace{p \rightarrow 1}^{s_{p} \cdot \pi} \Rightarrow \overbrace{1 \rightarrow 1}^{s_{p}}$ & $\pi \cdot s_{p}$ \\
\hline & $\overbrace{p \rightarrow p}^{\tau} \Rightarrow \overbrace{p \rightarrow 1}^{s_{p}}$ \\
\hline
\end{tabular}


Case $x=p^{-1}$. From $\pi\left(p^{-1}\right)=p, d_{m-1}=p$ and $d_{m}=p$ it follows that $\tau\left(p^{-1}\right)=2$. As the result, we have the following table.

\begin{tabular}{|c|c|}
\hline$\overbrace{p^{-1} \rightarrow p^{-1}}^{s_{p} \cdot \pi} \Rightarrow \overbrace{p^{-1} \rightarrow p}^{\pi} \overbrace{p^{-1} \rightarrow 2}^{\pi} \Rightarrow \overbrace{2 \rightarrow p}^{s_{p}}$ \\
\hline
\end{tabular}

ii) Let $d=2$ and $d_{m-1} \neq p$. That means that there exists the path of $p$ over $D$ such that $p \stackrel{r-1}{\longrightarrow} 1 \stackrel{r}{\rightarrow} 2 \stackrel{r+1}{\longrightarrow} x \Rightarrow \ldots \stackrel{w, m-1}{\longrightarrow} d_{m-1} \stackrel{m-1}{\longrightarrow} 1 \stackrel{m}{\rightarrow} 2$. The last means that $\sigma=\left[d_{r}, \ldots, d_{m-1}\right]_{S}$ satisfies conditions of the lemma. Based on induction assumption, the decomposition $D$ can be transformed into some decomposition $Y=\left(y_{1}, \ldots, y_{u}\right)$ such that $u \leq m, y_{u-1}=p$ and there is no $t \in \overline{1, u-2}$ such that $p=y_{t}$. Based on previous case, we can obtain $\tau$.

iii) Let $d \neq 2$. Note, that based on the lemma assumptions, we have the next path of the point 2 over $D$, namely $2 \stackrel{1}{\rightarrow} d_{1} \stackrel{1, t_{1}}{\longrightarrow} \ldots \stackrel{t_{2}, m-2}{\longrightarrow} d_{m-2} \stackrel{m-2}{\longrightarrow} 1 \stackrel{m-1}{\longrightarrow} 2 \stackrel{m}{\rightarrow} d_{m}$ for some natural numbers $t_{1}, t_{2}$. Suppose that $t_{1} \neq m-2$ and $t_{2}=1$. Then the permutation $\sigma=$ $\left[d_{t_{2}+1}, \ldots, d_{m-2}\right]$ satisfies conditions of the lemma. Based on induction assumption, the decomposition $D$ can be transformed into some decomposition $Y=\left(y_{1}, \ldots, y_{u}\right)$ such that $u \leq m$, $y_{1}=y_{u-2}$ and there is no $t \in \overline{2, u-3}$ such that $y_{1}=y_{t}$.

So, without loosing of generality, we can assume that $t_{1}=m-2$ and $t_{2}=1$. Consider different cases of $d_{m-1}$.

Case $d_{m-1}=p$. Construct $\tau$ as follows

$$
\tau=\left[t_{1}, \ldots, t_{m-2}\right]_{S}, \quad \text { where } t_{i}= \begin{cases}d_{1}, & \text { if } d_{i}=p \\ d_{i}, & \text { otherwise }\end{cases}
$$

From the construction of $\tau$ it follows that for every point $x, x \notin\left\{1,2, p, p^{-1}\right\}$, we have $\pi(x) \notin\left\{1,2, p, p^{-1}\right\}$. Therefore, $\left(s_{p} \cdot \pi\right)(x)=\pi(x)=\tau(x)=\left(\tau \cdot s_{p}\right)(x)$. Hence, it is enough to show that $\left(s_{p} \cdot \pi\right)(x)=\left(\tau \cdot s_{p}\right)(x)$ for every point $x$ from $\left\{1,2, p, p^{-1}\right\}$.

Subcase $x=1$. Note, that $d_{2}$ is the unique point in the decomposition of $\tau$. So, $\tau(1)=d_{2}$. As the result, we have the following table.

\begin{tabular}{|c|c|}
\hline$\overbrace{1 \rightarrow 2}^{s_{p} \cdot \pi} \Rightarrow \overbrace{2 \rightarrow d_{m}}^{s_{p}}$ & $\overbrace{1 \rightarrow d_{2}}^{\tau} \Rightarrow \overbrace{d_{2} \rightarrow d_{2}}^{s_{p}}$ \\
\hline
\end{tabular}

Subcase $x=2$. From $\pi(p)=d$ and substitution $p$ by $d_{1}$ it follows that there is the path of the point 2 in $\tau$, namely $2 \stackrel{1}{\rightarrow} d_{1} \stackrel{1, x}{\Longrightarrow} d_{1} \rightarrow \ldots \rightarrow d$, where $x$ is the first position of $p$ in $\pi$. As the result, we have the following table.

\begin{tabular}{|c|c|}
\hline$\overbrace{2 \rightarrow p}^{s_{p} \cdot \pi} \Rightarrow \overbrace{p \rightarrow d}^{\pi}$ & $\overbrace{2 \rightarrow d}^{\tau} \Rightarrow \overbrace{d \rightarrow d}^{s_{p}}$ \\
\hline
\end{tabular}

Subcase $x=p$. In this case, we have the following table.

\begin{tabular}{|c|c|}
\hline$\overbrace{p \rightarrow 1}^{s_{p} \cdot \pi} \Rightarrow \overbrace{1 \rightarrow 1}^{s_{p}}$ & $\pi \cdot s_{p}$ \\
\hline & $\overbrace{p \rightarrow p}^{\tau} \Rightarrow \overbrace{p \rightarrow 1}^{s_{p}}$ \\
\hline
\end{tabular}


Subcase $x=p^{-1}$. From $\pi\left(p^{-1}\right)=p, d_{m-1}=p$ and $d_{m}=p$ it follows that $\tau\left(p^{-1}\right)=2$. As the result, we have the following table.

\begin{tabular}{|c|c|}
\hline$\overbrace{p^{-1} \rightarrow p^{-1}}^{s_{p}} \cdot \pi$ & \multicolumn{1}{|c|}{$\pi \cdot \overbrace{p^{-1} \rightarrow p}^{\pi} \rightarrow \overbrace{p^{-1} \rightarrow 2}^{\tau} \Rightarrow \overbrace{2 \rightarrow p}^{s_{p}}$} \\
\hline
\end{tabular}

Case $d_{m_{1}} \neq p$. Construct $\tau$ as follows.

$$
\tau=\left[t_{1}, \ldots, t_{m-2}, t_{m-1}\right]_{S}, \quad \text { where } t_{i}= \begin{cases}d_{1}, & \text { if } d_{i}=p \\ d_{i}, & \text { otherwise }\end{cases}
$$

From the construction of $\tau$ it follows that for every point $x, x \notin\left\{1,2, p, p^{-1}\right\}$, we have $\pi(x) \notin\left\{1,2, p, p^{-1}\right\}$. Therefore, $\left(s_{p} \cdot \pi\right)(x)=\pi(x)=\tau(x)=\left(\tau \cdot s_{p}\right)(x)$. Hence, it is enough to show that $\left(s_{p} \cdot \pi\right)(x)=\left(\tau \cdot s_{p}\right)(x)$ for every point $x$ from $\left\{1,2, p, p^{-1}\right\}$.

Subcase $x=1$. Note, that $d_{2}$ is the unique point in the decomposition of $\tau$. So, $\tau(1)=d_{2}$. As the result, we have the following table.

$$
\begin{array}{|c|c|}
\hline \overbrace{1 \rightarrow 2}^{s_{p} \cdot \pi} \Rightarrow \overbrace{2 \rightarrow d_{m}}^{s_{p}} & \pi \cdot s_{p} \\
\hline \overbrace{1 \rightarrow d_{2}}^{\tau} \Rightarrow \overbrace{d_{2} \rightarrow d_{2}}^{s_{p}} \\
\hline
\end{array}
$$

Subcase $x=2$. From $\pi(p)=d$ and substitution $p$ by $d_{1}$ implies that there is the path of the point 1 in $\tau$, namely $1 \stackrel{1}{\rightarrow} d_{1} \stackrel{1, x}{\Longrightarrow} d_{1} \stackrel{\cdots}{\rightarrow} \stackrel{d}{\rightarrow}$, where $x$ is the first position of $p$ in $\pi$. As the result, we have the following table.

$$
\begin{array}{|c|c|}
\hline \overbrace{2 \rightarrow p}^{s_{p} \cdot \pi} \Rightarrow \overbrace{p \rightarrow d}^{\pi} & \overbrace{2 \rightarrow d}^{\tau} \Rightarrow \overbrace{d \rightarrow d}^{s_{p}} \\
\hline
\end{array}
$$

Subcase $x=p$. In this case, we have the following table.

\begin{tabular}{|c|c|}
\hline$\overbrace{p \rightarrow 1}^{s_{p} \cdot \pi} \Rightarrow \overbrace{1 \rightarrow 1}^{s_{p}}$ & $\pi \cdot s_{p}$ \\
\hline & $\overbrace{p \rightarrow p}^{\tau} \Rightarrow \overbrace{p \rightarrow 1}^{s_{p}}$ \\
\hline
\end{tabular}

Subcase $x=p^{-1}$. From $\pi\left(p^{-1}\right)=p$ it follows that there is the path of the point $p^{-1}$ in $\tau$, namely $p^{-1} \rightarrow \ldots \stackrel{x}{\rightarrow} d_{1} \stackrel{x, m-2}{\longrightarrow} d_{m-2} \stackrel{m-2}{\longrightarrow} 1 \stackrel{m-1}{\longrightarrow} 2$, where $x$ is the last position of $p$ in $D$. As the result, we have the following table.

\begin{tabular}{|c|c|}
\hline$\overbrace{p^{-1} \rightarrow p^{-1}}^{s_{p} \cdot \pi} \Rightarrow \overbrace{p^{-1} \rightarrow p}^{s_{p}} \rightarrow \overbrace{p^{-1} \rightarrow 2}^{\pi} \Rightarrow \overbrace{2 \rightarrow p}^{s_{p}}$ \\
\hline
\end{tabular}

Let $d \neq 2$. Then there exists the natural numbers $x, y$ and path of $d=d_{y}$ in $\pi$ over $D$ such that $d \stackrel{y}{\rightarrow} 1 \stackrel{y+1}{\longrightarrow} 2 \stackrel{y+2}{\longrightarrow} d_{y+2} \Rightarrow \ldots \stackrel{x, m-2}{\longrightarrow} d_{m-2} \stackrel{m-2}{\longrightarrow} 1 \stackrel{m-1}{\longrightarrow} 2 \stackrel{m}{\rightarrow} d_{m}$. The last means that $\operatorname{Tr}(d, \pi, D)=\left(y, t_{1}, r_{1}, \ldots, t_{u}, r_{u}, m\right)$ for some natural numbers $t_{1}, r_{1}, \ldots, t_{u}, r_{u} \in \overline{y+2, m-2}$, $u \geq 0$. Let $\pi_{k}=\left[d_{t_{k}-1}, d_{t_{k}}, \ldots, d_{r_{k+1}}\right]$ for every $k \in \overline{1, u}$. Note, that $\pi_{k}$ satisfies assumption of the lemma and the length of its decomposition is less than $m$. Then, based on induction assumption, for every natural $p, p \geq 3$, we have $s_{p} \cdot \pi_{k}=\tau_{k} \cdot s_{p}$ for some $\tau_{k}$, which length of 
the decomposition is less or equal to $r_{k}-t_{k}+2$. Let $v=\left[d_{1}, \ldots, d_{y-1}\right]_{S}$ and $\mu=\left[d_{m-1}, d_{m}\right]_{S}$. Then $\pi=v \cdot s_{d} \cdot \pi_{1} \cdot \ldots \cdot \pi_{u} \cdot \mu=v \cdot \tau_{1} \cdot s_{d} \cdot \pi_{2} \cdot \ldots \cdot \pi_{u} \cdot \mu=\ldots=v \cdot \tau_{1} \cdot \ldots \cdot \tau_{u} \cdot s_{d} \cdot \mu$.

Note, that transformation from $\pi_{k}$ to $\tau_{k}$ does not introduce points $d_{2}, d_{m}$, because they are unique represented in $D$ and they are not represented in $\pi_{k}, k \in \overline{1, u}$; there are no $d$ in the decomposition $Y$ of $\pi$, which is the concatenation of considered decompositions $v, \tau_{1}, \ldots, \tau_{u},[d]_{S}, \mu, \pi(d)=d_{m}$.

As the result, from Lemma 4 it follows the proof.

\subsection{Multiplication from the right}

Let $\pi \in A$ be a permutation with the decomposition $D=\left(d_{1}, \ldots, d_{m}\right), m \geq 3$, over $S$. Note, that if the point 2 is fixed in $\pi$ and has trivial path in $\pi$ over $D$, then, based on Proposition 2 , the path of the point 2 over $D$ is the following $2 \stackrel{1}{\rightarrow} d_{1} \stackrel{d_{1}, d_{m-1}}{\longrightarrow} d_{m-1} \stackrel{m-1}{\longrightarrow} 1 \stackrel{m}{\rightarrow} 2$.

Lemma 6. Let $\pi \in A$ be some permutation with the decomposition $D=\left(d_{1}, d_{2}, \ldots, d_{m}\right)$, $m \geq 3,2$ is fixed in $\pi$ over $D$ with trivial path, $d=\pi\left(d_{1}\right)$. Then for every $p \notin\left\{d_{1}, \ldots, d_{m}\right\}$, $p \geq 3$, we have $\pi \cdot s_{p}=s_{p} \cdot \tau$, where

$$
\tau= \begin{cases}{\left[d_{2}, \ldots, d_{m-1}, d_{m}, d\right]_{S},} & \text { if } d \neq 1, \\ {\left[d_{2}, \ldots, d_{m-1}, d_{m}\right]_{S},} & \text { if } d=1 .\end{cases}
$$

Proof. Let $\sigma=\left[d_{2}, \ldots, d_{m}\right]_{S}$. Consider cases $d \neq 1$ and $d=1$ independently.

Let $d \neq 1$. From the construction of $\tau$ it follows that for every point $x, x \notin\left\{1,2, p, d_{1}\right\}$, we have $\pi(x) \notin\left\{1,2, p, d_{1}\right\}$. Therefore, $\left(\pi \cdot s_{p}\right)(x)=\pi(x)=\tau(x)=\left(s_{p} \cdot \tau\right)(x)$. Hence, it is enough to show that $\left(\pi \cdot s_{p}\right)(x)=\left(s_{p} \cdot \tau\right)(x)$ for every point $x$ from $\left\{1,2, p, d_{1}\right\}$.

Case $x=1$. Note, that $\pi(1) \neq 2$. So, $\pi(1)$ is some number from $\left\{d_{2}, d_{3}, \ldots, d_{m}\right\}$ or equal 1 . If $\pi(1) \neq 1$, then $\pi(1)=\sigma(2)$. So, $\tau(2)=\pi(1)$. If $\pi(1)=1$, then $\sigma(2)=1$. So, $\tau(2)=2$.

As the result, we have the following table.

\begin{tabular}{|l|c|c|}
\hline & $\pi \cdot s_{p}$ & $s_{p} \cdot \tau$ \\
\hline$\pi(1) \neq 1$ & $\overbrace{1 \rightarrow \pi(1)}^{\pi} \Rightarrow \overbrace{\pi(1) \rightarrow \pi(1)}^{s_{p}}$ & $\overbrace{1 \rightarrow 2}^{s_{p}} \Rightarrow \overbrace{2 \rightarrow \pi(1)}^{\tau}$ \\
$\overbrace{1 \rightarrow 1}^{s_{p}} \Rightarrow \overbrace{1 \rightarrow 2}^{s_{p}}$ & $\overbrace{1 \rightarrow 2}^{\tau} \Rightarrow \overbrace{2 \rightarrow 2}^{\tau}$ \\
\hline
\end{tabular}

Case $x=2$. In this case, we have the following table.

\begin{tabular}{|c|c|}
\hline$\pi \cdot s_{p}$ & $s_{p} \cdot \tau$ \\
\hline$\overbrace{2 \rightarrow 2}^{\pi} \Rightarrow \overbrace{2 \rightarrow p}^{s_{p}}$ & $\overbrace{2 \rightarrow p}^{s_{p}} \Rightarrow \overbrace{p \rightarrow p}^{\tau}$ \\
\hline
\end{tabular}

Case $x=p$. Note, that $\sigma(1)=\pi\left(d_{1}\right)=d$. So, $\tau(1)=1$. As the result, we have the following table.

\begin{tabular}{|c|c|}
\hline$\pi \cdot s_{p}$ & $s_{p} \cdot \tau$ \\
\hline$\overbrace{p \rightarrow p}^{\pi} \Rightarrow \overbrace{p \rightarrow 1}^{s_{p}}$ & $\overbrace{p \rightarrow 1}^{s_{p}} \Rightarrow \overbrace{1 \rightarrow 1}^{\tau}$ \\
\hline
\end{tabular}


Case $x=d_{1}$. Note, that $d_{1}$ has unique representation in the decomposition of $\tau$ on the $(m-2)$ th position. So, $\tau\left(d_{1}\right)=d$. As the result, we have the following table.

\begin{tabular}{|c|c|}
\hline$\pi \cdot s_{p}$ & $s_{p} \cdot \tau$ \\
\hline$\overbrace{d_{1} \rightarrow d}^{\pi} \Rightarrow \overbrace{d \rightarrow d}^{s_{p}}$ & $\overbrace{d_{1} \rightarrow d_{1}}^{s_{p}} \Rightarrow \overbrace{d_{1} \rightarrow d}^{\pi}$ \\
\hline
\end{tabular}

Let $d=1$. From the construction of $\tau$ it follows that for every point $x, x \notin\left\{1,2, p, d_{1}\right\}$, we have $\pi(x) \notin\left\{1,2, p, d_{1}\right\}$. Therefore, $\left(\pi \cdot s_{p}\right)(x)=\pi(x)=\tau(x)=\left(s_{p} \cdot \tau\right)(x)$. Hence, it is enough to show that $\left(\pi \cdot s_{p}\right)(x)=\left(s_{p} \cdot \tau\right)(x)$ for every point $x$ from $\left\{1,2, p, d_{1}\right\}$.

Case $x=1$. Note, that $\pi(1) \neq 1$ and $\tau(2)=\pi(1)$. As the result, we have the following table.

\begin{tabular}{|c|c|}
\hline$\pi \cdot s_{p}$ & $s_{p} \cdot \tau$ \\
\hline$\overbrace{1 \rightarrow \pi(1)}^{\pi} \Rightarrow \overbrace{\pi(1) \rightarrow \pi(1)}^{s_{p}}$ & $\overbrace{1 \rightarrow 2}^{s_{p}} \Rightarrow \overbrace{2 \rightarrow \pi(1)}^{\tau}$ \\
\hline
\end{tabular}

Case $x=2$. In this case, we have the following table.

\begin{tabular}{|c|c|}
\hline$\pi \cdot s_{p}$ & $s_{p} \cdot \tau$ \\
\hline$\overbrace{2 \rightarrow 2}^{\pi} \Rightarrow \overbrace{2 \rightarrow p}^{s_{p}}$ & $\overbrace{2 \rightarrow p}^{s_{p}} \Rightarrow \overbrace{p \rightarrow p}^{\tau}$ \\
\hline
\end{tabular}

Case $x=p$. Note, that $\tau(1)=\pi\left(d_{1}\right)=1$. As the result, we have the following table.

\begin{tabular}{|c|c|}
\hline$\pi \cdot s_{p}$ & $s_{p} \cdot \tau$ \\
\hline$\overbrace{p \rightarrow p}^{\pi} \Rightarrow \overbrace{p \rightarrow 1}^{s_{p}}$ & $\overbrace{p \rightarrow 1}^{s_{p}} \Rightarrow \overbrace{1 \rightarrow 1}^{\tau}$ \\
\hline
\end{tabular}

The proof is complete.

Lemma 7. Let $\pi \in A$ be a permutation with decomposition $D=\left(d_{1}, \ldots, d_{m}\right), m \geq 3,2$ is fixed point in $\pi$ over $D$ with trivial path and $\pi\left(d_{1}\right)=d_{3}$, which is not in $\left(d_{4}, \ldots, d_{m}\right)$. Then for every natural number $p, p \geq 3$, there exists $\tau \in A$ such that $\pi \cdot s_{p}=s_{p} \cdot \tau$ and $\tau$ has a decomposition $\left(t_{1}, \ldots, t_{l}\right)$, where $l \leq m$ and $p \notin\left\{t_{1}, \ldots, t_{l}\right\}$.

Proof. Note, that if $p \notin\left\{d_{1}, \ldots, d_{m}\right\}$, then the statement holds by Lemma 6 .

Let $p \in\left\{d_{1}, \ldots, d_{m}\right\}$. Consider seven different cases:

1) $d_{1}=p$;

2) $d_{1} \neq p, d_{2}, d_{3} \neq p$;

3) $d_{1} \neq p, d_{2}=p$ and $d_{3} \neq p$;

4) $d_{1} \neq p, d_{2} \neq p, d_{3}=p$ and $\pi(p) \neq 1$;

5) $d_{1} \neq p, d_{2}, d_{3}=p$ and $\pi(p) \neq 1$;

6) $d_{1} \neq p, d_{2} \neq p, d_{3}=p$ and $\pi(p)=1$;

7) $d_{1} \neq p, d_{2}, d_{3}=p$ and $\pi(p)=1$. 
1) Let $d_{1}=d_{m-1}=p$. Consider two cases.

(a) If $d_{m} \neq p$, then based on Proposition 1 we have

$$
\begin{aligned}
\pi \cdot s_{p}=\left[p, d_{2}, \ldots, d_{m-2}, p, d_{m}\right]_{S} \cdot[p]_{S} & =[p]_{S} \cdot\left[d_{2}, \ldots, d_{m-2}, d_{m}, d_{m}, p, p, p\right]_{S} \\
& =[p]_{S} \cdot\left[d_{2}, \ldots, d_{m-2}, d_{m}, d_{m}\right]_{S}=s_{p} \cdot \tau,
\end{aligned}
$$

where the length of the decomposition of $\tau$ is less than the length of $D$ at least by 1 .

(b) If $d_{m}=p$, then based on Proposition 1 we have

$$
\begin{aligned}
\pi \cdot s_{p}=\left[p, d_{2}, \ldots, d_{m-2}, p, p\right]_{S} \cdot[p]_{S} & =[p]_{S} \cdot\left[d_{2}, \ldots, d_{m-2}, p, p, p\right]_{S} \\
& =[p]_{S} \cdot\left[d_{2}, \ldots, d_{m-2}\right]_{S}=s_{p} \cdot \tau,
\end{aligned}
$$

where the length of the decomposition of $\tau$ is less than the length of $D$ at least by 3 .

2) Let $d_{2} \neq p, d_{3} \neq p$. Construct $\tau$ as follows

$$
\tau=\left[t_{2}, t_{3}, \ldots, t_{m-1}, t_{m}, d_{3}\right]_{S}, \quad \text { where } d_{i}= \begin{cases}d_{3}, & \text { if } d_{i}=p, \\ d_{i}, & \text { otherwise. }\end{cases}
$$

Let $\sigma=\left[t_{2}, \ldots, t_{m}\right]_{S}$. From the construction of $\tau$ it follows that for every point $x, x \notin\left\{1,2, p, d_{3}\right\}$, we have $\pi(x) \notin\left\{1,2, p, d_{3}\right\}$. Therefore, $\left(\pi \cdot s_{p}\right)(x)=\pi(x)=\tau(x)=\left(s_{p} \cdot \tau\right)(x)$. Hence, it is enough to show that $\left(\pi \cdot s_{p}\right)(x)=\left(s_{p} \cdot \tau\right)(x)$ for every point $x$ from $\left\{1,2, p, d_{3}\right\}$.

Case $x=1$. Note, that:

(a) if $\pi(1)=1$, then $\overbrace{2 \rightarrow 1}^{\sigma} \Rightarrow \overbrace{1 \rightarrow 2}^{\left(1,2, d_{3}\right)}$, which means that $\tau(2)=2$;

(b) if $\pi(1)=p$, then $\overbrace{2 \rightarrow d_{3}}^{\sigma} \Rightarrow \overbrace{d_{3} \rightarrow 1}^{\left(1,2, d_{3}\right)}$, which means that $\tau(2)=1$;

(c) if $\pi(1) \neq 1, \pi(1) \neq p$, then $\tau(2)=\pi(1)$.

As the result, we have the following table.

\begin{tabular}{|c|c|c|}
\hline$\pi(1)=1$ & $\overbrace{1 \rightarrow 1}^{\pi} \Rightarrow \overbrace{1 \rightarrow 2}^{s_{1 \rightarrow}}$ & $\overbrace{1 \rightarrow 2}^{s_{p}} \Rightarrow \overbrace{2 \rightarrow 2}^{s_{p}}$ \\
$\pi(1)=p$ & $\overbrace{1 \rightarrow p}^{s_{p}} \Rightarrow \overbrace{p \rightarrow 1}^{s_{p}}$ \\
$\pi(1) \neq 1, p$ & $\overbrace{1 \rightarrow \pi(1)}^{\pi} \Rightarrow \overbrace{\pi(1) \rightarrow \pi(1)}^{s_{p}}$ & $\overbrace{1 \rightarrow 2}^{s_{p}} \Rightarrow \overbrace{2 \rightarrow 1}^{s_{p}}$ \\
$\overbrace{1 \rightarrow 2}^{s_{p}} \Rightarrow \overbrace{2 \rightarrow \pi(1)}^{\tau}$ \\
\hline
\end{tabular}

Case $x=2$. Note, that $\tau(p)=p$. As the result, we have the following table.

\begin{tabular}{|c|c|}
\hline$\pi \cdot s_{p}$ & $s_{p} \cdot \tau$ \\
\hline$\overbrace{2 \rightarrow 2}^{\pi} \Rightarrow \overbrace{2 \rightarrow p}^{s_{p}}$ & $\overbrace{1 \rightarrow 2}^{s_{p}} \Rightarrow \overbrace{2 \rightarrow p}^{\tau}$ \\
\hline
\end{tabular}

Case $x=p$. Note, that:

(a) if $\pi(p)=1$, then $\sigma(1)=\left[t_{2}, \ldots, t_{m}\right]_{S}=1$, so $\overbrace{1 \rightarrow 1}^{\sigma} \Rightarrow \overbrace{1 \rightarrow 2}^{\left(1,2, d_{3}\right)}$, which means that $\tau(1)=2$;

(b) if $\pi(p)=p$, then $\overbrace{1 \rightarrow d_{3}}^{\sigma} \Rightarrow \overbrace{d_{3} \rightarrow 1}^{\left(1,2, d_{3}\right)}$, which means that $\tau(1)=1$;

(c) if $\pi(p) \neq 1, \pi(p) \neq p$, then $\tau(1)=\pi(p)$, based on the construction of $\tau$. 
As the result, we have the following table.

\begin{tabular}{|c|c|c|}
\hline & $\pi \cdot s_{p}$ & $s_{p} \cdot \tau$ \\
\hline$\pi(p)=1$ & $\overbrace{p \rightarrow 1}^{\pi} \Rightarrow \overbrace{1 \rightarrow 2}^{s_{p}}$ & $\overbrace{p \rightarrow 1}^{s_{p}} \Rightarrow \overbrace{1 \rightarrow 2}^{\tau}$ \\
\hline$\pi(p)=p$ & $\overbrace{p \rightarrow p}^{p} \Rightarrow \overbrace{p \rightarrow 1}$ & $\overparen{p \rightarrow 1} \Rightarrow 1 \rightarrow 1$ \\
\hline$\pi(p) \neq 1, p$ & $\overbrace{p \rightarrow \pi(p)}^{\Rightarrow} \Rightarrow \overbrace{\pi(p) \rightarrow \pi(p)}$ & $\overbrace{p \rightarrow 1} \Rightarrow \overbrace{1 \rightarrow \pi(p)}$ \\
\hline
\end{tabular}

Case $x=d_{3}$. Note, that:

(a) if $\pi\left(d_{3}\right)=1$, then $\overbrace{d_{3} \rightarrow 1}^{\sigma} \Rightarrow \overbrace{1 \rightarrow 2}^{\left(1,2, d_{3}\right)}$, which means that $\tau\left(d_{3}\right)=2$;

(b) if $\pi\left(d_{3}\right)=p$, then $\overbrace{d_{3} \rightarrow d_{3}}^{\sigma} \Rightarrow \overbrace{\left.d_{3} \rightarrow 1,1, d_{3}\right)}^{(1, d}$, which means that $\tau\left(d_{3}\right)=1$;

(c) if $\pi\left(d_{3}\right) \neq 1, \pi\left(d_{3}\right) \neq p$, then $\tau\left(d_{3}\right)=\pi\left(d_{3}\right)$.

As the result, we have the following table.

\begin{tabular}{|c|c|c|}
\hline & $\pi \cdot s_{p}$ & $s_{p} \cdot \tau$ \\
\hline$\pi\left(d_{3}\right)=1$ & $\begin{array}{c}\overbrace{d_{3} \rightarrow 1}^{\pi} \Rightarrow \overbrace{1 \rightarrow 2}^{s_{p}} \\
\overbrace{}^{s_{p}}\end{array}$ & $\overbrace{d_{3} \rightarrow d_{3}}^{s_{p} p} \Rightarrow \overbrace{d_{3} \rightarrow 2}^{\tau}$ \\
\hline$\pi\left(d_{3}\right)=p$ & $\overbrace{}^{\overbrace{d_{3}} \rightarrow p} \Rightarrow \overbrace{p \rightarrow 1} \overbrace{s_{p}}^{s_{p}}$ & $\overbrace{d_{3}}^{s_{p}} \rightarrow d_{d_{3}} \Rightarrow \overbrace{d_{3}}^{\tau} \rightarrow 1$ \\
\hline$\pi\left(d_{3}\right) \neq 1, p$ & 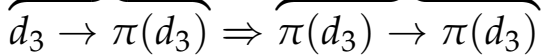 & $\overparen{d_{3} \rightarrow d_{3}} \Rightarrow \overparen{d_{3} \rightarrow \pi\left(d_{3}\right)}$ \\
\hline
\end{tabular}

3) Let $d_{2}=p$ and $d_{3} \neq p$. Construct $\tau$ as follows

$$
\tau=\left[t_{3}, \ldots, t_{m-1}, t_{m}, d_{3}\right]_{S}, \quad \text { where } t_{i}= \begin{cases}d_{3}, & \text { if } d_{i}=p \\ d_{i}, & \text { otherwise }\end{cases}
$$

Let $\sigma=\left[t_{3}, \ldots, t_{m}\right]_{S}$. From the construction of $\tau$ it follows that for every point $x, x \notin\left\{1,2, p, d_{3}\right\}$, we have $\pi(x) \notin\left\{1,2, p, d_{3}\right\}$. Therefore, $\left(\pi \cdot s_{p}\right)(x)=\pi(x)=\tau(x)=\left(s_{p} \cdot \tau\right)(x)$. Hence, it is enough to show that $\left(\pi \cdot s_{p}\right)(x)=\left(s_{p} \cdot \tau\right)(x)$ for every point $x$ from $\left\{1,2, p, d_{3}\right\}$.

Case $x=1$. Note, that:

(a) if $\pi(1)=1$, then $\sigma(2)=\pi(1)=1$, so, $\tau(2)=\left[d_{3}\right]_{S}(1)=2$;

(b) if $\pi(1)=p$, then $\sigma(2)=d_{3}$, so, $\tau(2)=\left[d_{3}\right]_{S}\left(d_{3}\right)=1$;

(c) if $\pi(1) \neq 1, \pi(1) \neq p$, then $\tau(2)=\pi(1)$.

As the result, we have the following table.

\begin{tabular}{|c|c|c|}
\hline & $\pi \cdot s_{p}$ & $s_{p} \cdot \tau$ \\
\hline$\pi(1)=1$ & $\overbrace{1 \rightarrow 1}^{\pi} \Rightarrow \overbrace{1 \rightarrow 2}^{s_{p}}$ & $\overbrace{1 \rightarrow 2}^{s_{p}} \Rightarrow \overbrace{2 \rightarrow 2}^{\tau}$ \\
\hline$\pi(1)=p$ & $\overparen{1 \rightarrow p} \Rightarrow \overparen{p \rightarrow 1}$ & $\overparen{1 \rightarrow 2} \Rightarrow \overparen{2 \rightarrow 1}$ \\
\hline & & $s_{p} \quad \tau$ \\
\hline$\pi(1) \neq 1, p$ & $\overparen{1 \rightarrow \pi(1)} \Rightarrow \overparen{\pi(1) \rightarrow \pi(1)}$ & $\overbrace{1 \rightarrow 2} \Rightarrow \overbrace{2 \rightarrow \pi(1)}$ \\
\hline
\end{tabular}


Case $x=2$. Note, that $\tau(p)=p$. As the result, we have the following table.

\begin{tabular}{|c|c|}
\hline$\pi \cdot s_{p}$ & $s_{p} \cdot \tau$ \\
\hline$\overbrace{2 \rightarrow 2}^{\pi} \Rightarrow \overbrace{2 \rightarrow p}^{s_{p}}$ & $\overbrace{1 \rightarrow 2}^{s_{p}} \Rightarrow \overbrace{2 \rightarrow p}^{\tau}$ \\
\hline
\end{tabular}

Case $x=p$. Note, that:

(a) if $\pi(p)=1$, then $\sigma(1)=\pi\left(d_{2}\right)=\pi(p)=1$, so $\overbrace{1 \rightarrow 1}^{\sigma} \Rightarrow \overbrace{1 \rightarrow 2}^{\left(1,2, d_{3}\right)}$, which means that $\tau(1)=2$;

(b) if $\pi(p)=p$, then $\sigma(1)=d_{3}$, so $\overbrace{1 \rightarrow d_{3}}^{\sigma} \Rightarrow \overbrace{d_{3} \rightarrow 1}^{\left(1,2, d_{3}\right)}$, which means that $\tau(1)=1$;

(c) if $\pi(p) \neq 1, \pi(p) \neq p$, then $\tau(1)=\pi(p)$, based on the construction of $\tau$.

As the result, we have the following table.

\begin{tabular}{|c|c|c|}
\hline$\pi(p)=1$ & $\overbrace{p \rightarrow 1}^{\pi} \Rightarrow \overbrace{1 \rightarrow 2}^{s_{p}}$ & $\overbrace{p \rightarrow 1}^{s_{p \rightarrow}} \Rightarrow \overbrace{1 \rightarrow 2}^{s_{p}} \cdot \tau$ \\
$\pi(p)=p$ & $\overbrace{p \rightarrow p}^{s_{p}} \Rightarrow \overbrace{p \rightarrow 1}^{s_{p}}$ & $\overbrace{p \rightarrow 1}^{s_{p}} \Rightarrow \overbrace{1 \rightarrow 1}^{\tau}$ \\
$\pi(p) \neq 1, p$ & $\overbrace{p \rightarrow \pi(p)}^{\pi} \Rightarrow \overbrace{\pi(p) \rightarrow \pi(p)}^{s_{p}}$ & $\overbrace{p \rightarrow 1}^{s_{p}} \Rightarrow \overbrace{1 \rightarrow \pi(p)}^{\tau}$ \\
\hline
\end{tabular}

Case $x=d_{3}$. Note, that:

(a) if $\pi\left(d_{3}\right)=1$, then $\overbrace{d_{3} \rightarrow 1}^{\sigma} \Rightarrow \overbrace{1 \rightarrow 2}^{\left(1,2, d_{3}\right)}$, which means that $\tau\left(d_{3}\right)=2$;

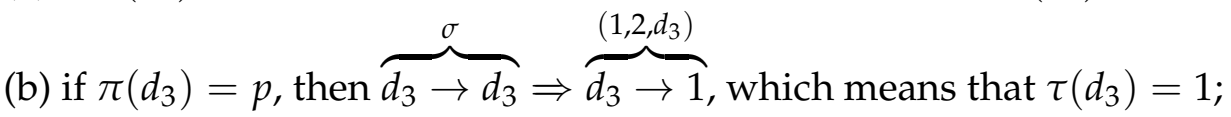

(c) if $\pi\left(d_{3}\right) \neq 1, \pi\left(d_{3}\right) \neq p$, then $\tau\left(d_{3}\right)=\pi\left(d_{3}\right)$.

As the result, we have the following table.

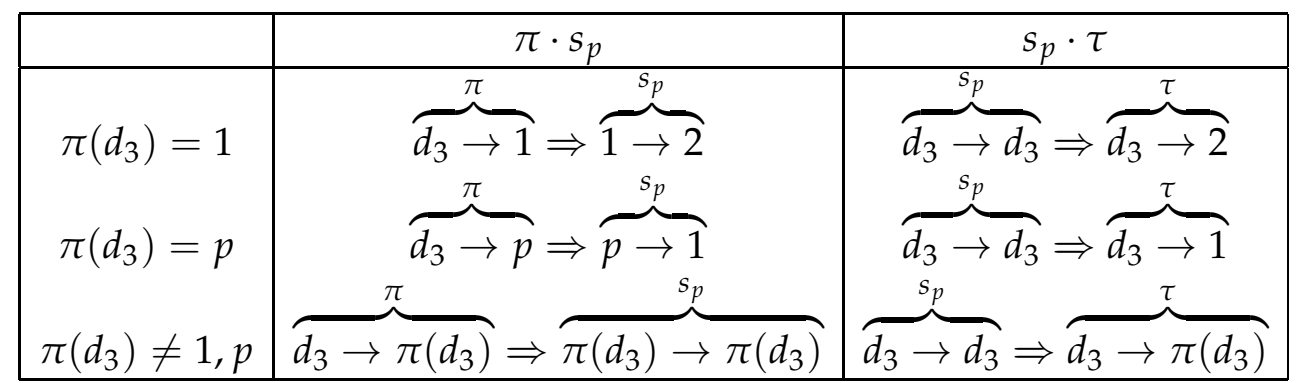

4) Let $d_{2} \neq p, d_{3}=p$ and $d=\pi(p) \neq 1$. Note, that $d_{3}$ is the unique representation of $p$ in $D$. Construct $\tau$ as follows $\tau=\left[d_{2}, d_{1}, d_{4}, d_{5}, \ldots, d_{m-1}, d_{m}, d\right]_{S}$.

that for every point $x, x \notin\left\{1,2, p, d_{1}\right\}$, we have $\pi(x) \notin\left\{1,2, p, d_{1}\right\}$. Therefore, $\left(\pi \cdot s_{p}\right)(x)=$ $\pi(x)=\tau(x)=\left(s_{p} \cdot \tau\right)(x)$. Hence, it is enough to show that $\left(\pi \cdot s_{p}\right)(x)=\left(s_{p} \cdot \tau\right)(x)$ for every point $x$ from $\left\{1,2, p, d_{1}\right\}$.

Case $x=1$. Note, that:

(a) if $\pi(1)=1$, then $\sigma(2)=\pi(1)=1$, so, $\tau(2)=[d]_{S}(1)=2$;

(b) if $\pi(1) \neq 1$, then $\overbrace{2 \rightarrow \pi(1)}^{\sigma} \Rightarrow \overbrace{\pi(1) \rightarrow \pi(1)}^{(1,2, d)}$, which means that $\tau(2)=\pi(1)$. 
As the result, we have the following table.

\begin{tabular}{|l|c|c|}
\hline & $\pi \cdot s_{p}$ & $\overbrace{1 \rightarrow 1}^{\pi} \Rightarrow \overbrace{1 \rightarrow 2}^{s_{p}}$ \\
$\pi(1) \neq 1$ & $\overbrace{1 \rightarrow \pi(1)}^{s_{p} \cdot \tau}$ & $\overbrace{1 \rightarrow 2}^{s_{p}} \Rightarrow \overbrace{2 \rightarrow 2}^{\tau}$ \\
$\overbrace{\pi(1) \rightarrow \pi(1)}^{s_{p}}$ & $\overbrace{1 \rightarrow 2}^{s_{p}} \Rightarrow \overbrace{2 \rightarrow \pi(1)}^{\tau}$ \\
\hline
\end{tabular}

Case $x=2$. Note, that $\tau(p)=p$. As the result, we have the following table.

\begin{tabular}{|c|c|}
\hline$\pi \cdot s_{p}$ & $s_{p} \cdot \tau$ \\
\hline$\overbrace{2 \rightarrow 2}^{\pi} \Rightarrow \overbrace{2 \rightarrow p}^{s_{p}}$ & $\overbrace{1 \rightarrow 2}^{s_{p}} \Rightarrow \overbrace{2 \rightarrow p}^{\tau}$ \\
\hline
\end{tabular}

Case $x=p$. Note, that there is the path of the point 1 over the decomposition of $\tau$, namely $1 \stackrel{1}{\rightarrow} 2 \stackrel{2}{\rightarrow} d_{1} \stackrel{2, m-2}{\longrightarrow} d_{m-1} \stackrel{m-2}{\longrightarrow} 1 \stackrel{m-1}{\longrightarrow} 2 \stackrel{m}{\rightarrow} d$, which means that $\tau(1)=d$. As the result, we have the following table.

$$
\begin{array}{|c|c|}
\hline \pi \cdot s_{p} & s_{p} \cdot \tau \\
\hline \overbrace{p \rightarrow d}^{\pi} \Rightarrow \overbrace{d \rightarrow d}^{s_{p}} & \overbrace{p \rightarrow 1}^{s_{p}} \Rightarrow \overbrace{1 \rightarrow d}^{\tau} \\
\hline
\end{array}
$$

Case $x=d_{1}$. Note, that in this case $\sigma\left(d_{1}\right)=\pi\left(d_{3}\right)=d$, which means that $\tau\left(d_{1}\right)=[d]_{S}(d)=1$. As the result, we have the following table.

\begin{tabular}{|c|c|}
\hline$\pi \cdot s_{p}$ & $s_{p} \cdot \tau$ \\
\hline$\overbrace{d_{1} \rightarrow p}^{\pi} \Rightarrow \overbrace{p \rightarrow 1}^{s_{p}}$ & $\overbrace{d_{1} \rightarrow d_{1}}^{s_{p}} \Rightarrow \overbrace{d_{1} \rightarrow 1}^{\tau}$ \\
\hline
\end{tabular}

5) Let $d_{2}=p, d_{3}=p$ and $d=\pi(p) \neq 1$. Note, that in this case $d_{2}, d_{3}$ are unique representations of $p$ in $D$. Construct $\tau$ as follows

$$
\tau=\left[d_{1}, d_{1}, d_{4}, d_{5}, \ldots, d_{m-1}, d_{m}, d\right]_{S}
$$

Let $\sigma=\left[d_{1}, d_{4}, d_{5}, \ldots, d_{m-1}, d_{m}\right]_{S}$. From the construction of $\tau$ it follows that for every point $x, x \notin\left\{1,2, p, d_{1}\right\}$, we have $\pi(x) \notin\left\{1,2, p, d_{1}\right\}$. Therefore, $\left(\pi \cdot s_{p}\right)(x)=\pi(x)=\tau(x)=$ $\left(s_{p} \cdot \tau\right)(x)$. Hence, it is enough to show that $\left(\pi \cdot s_{p}\right)(x)=\left(s_{p} \cdot \tau\right)(x)$ for every point $x$ from $\left\{1,2, p, d_{1}\right\}$.

Case $x=1$. Note, that:

(a) if $\pi(1)=1$, then $\sigma(2)=\pi(1)=1$, so, $\tau(2)=[d]_{S}(1)=2$;

(b) if $\pi(1) \neq 1$, then $\overbrace{2 \rightarrow \pi(1)}^{\sigma} \Rightarrow \overbrace{\pi(1) \rightarrow \pi(1)}^{(1,2, d)}$, which means that $\tau(2)=\pi(1)$.

As the result, we have the following table.

\begin{tabular}{|l|c|c|}
\hline & $\pi \cdot s_{p}$ & $s_{p} \cdot \tau$ \\
\hline$\pi(1)=1$ & $\overbrace{1 \rightarrow 1}^{\pi} \Rightarrow \overbrace{1 \rightarrow 2}^{s_{p}}$ & $\overbrace{1 \rightarrow 2}^{s_{p}} \Rightarrow \overbrace{2 \rightarrow 2}^{\tau}$ \\
$\overbrace{1 \rightarrow \pi(1)}^{s_{p}} \Rightarrow \overbrace{\pi(1) \rightarrow \pi(1)}^{s_{p}}$ & $\overbrace{1 \rightarrow 2}^{s_{p}} \Rightarrow \overbrace{2 \rightarrow \pi(1)}^{\tau}$ \\
\hline
\end{tabular}


Case $x=2$. Note, that $\tau(p)=p$. As the result, we have the following table.

\begin{tabular}{|c|c|}
\hline$\pi \cdot s_{p}$ & $s_{p} \cdot \tau$ \\
\hline$\overbrace{2 \rightarrow 2}^{\pi} \Rightarrow \overbrace{2 \rightarrow p}^{s_{p}}$ & $\overbrace{1 \rightarrow 2}^{s_{p}} \Rightarrow \overbrace{2 \rightarrow p}^{\tau}$ \\
\hline
\end{tabular}

Case $x=p$. Note, that there is the path of the point 1 over the decomposition of $\tau$, namely $1 \stackrel{1}{\rightarrow} 2 \stackrel{2}{\rightarrow} d_{1} \stackrel{2, m-2}{\longrightarrow} d_{m-1} \stackrel{m-2}{\longrightarrow} 1 \stackrel{m-1}{\longrightarrow} 2 \stackrel{m}{\rightarrow} d$, which means that $\tau(1)=d$. As the result, we have the following table.

$$
\begin{array}{|c|c|}
\hline \pi \cdot s_{p} & s_{p} \cdot \tau \\
\hline \overbrace{p \rightarrow d}^{\pi} \Rightarrow \overbrace{d \rightarrow d}^{s_{p}} & \overbrace{p \rightarrow 1}^{s_{p}} \Rightarrow \overbrace{1 \rightarrow d}^{\tau} \\
\hline
\end{array}
$$

Case $x=d_{1}$. Note, that in this case $\sigma\left(d_{1}\right)=\pi\left(d_{2}\right)=d$, which means that $\tau\left(d_{1}\right)=[d]_{S}(d)=1$. As the result, we have the following table.

\begin{tabular}{|c|c|}
\hline$\pi \cdot s_{p}$ & $s_{p} \cdot \tau$ \\
\hline$\overbrace{d_{1} \rightarrow p}^{\pi} \Rightarrow \overbrace{p \rightarrow 1}^{s_{p}}$ & $\overbrace{d_{1} \rightarrow d_{1}}^{s_{p}} \Rightarrow \overbrace{d_{1} \rightarrow 1}^{\tau}$ \\
\hline
\end{tabular}

6) Let $d_{2} \neq p, d_{3}=p$ and $\pi(p)=1$. Note, that in this case $d_{3}$ is the unique representation of $p$ in $D$. Construct $\tau$ as follows

$$
\tau=\left[d_{2}, d_{1}, d_{4}, d_{5}, \ldots, d_{m-1}, d_{m}\right]_{S}
$$

From the construction of $\tau$ it follows that for every point $x, x \notin\left\{1,2, p, d_{1}\right\}$, we have $\pi(x) \notin$ $\left\{1,2, p, d_{1}\right\}$. Therefore, $\left(\pi \cdot s_{p}\right)(x)=\pi(x)=\tau(x)=\left(s_{p} \cdot \tau\right)(x)$. Hence, it is enough to show that $\left(\pi \cdot s_{p}\right)(x)=\left(s_{p} \cdot \tau\right)(x)$ for every point $x$ from $\left\{1,2, p, d_{1}\right\}$.

Case $x=1$. Note, that $\tau(2)=\pi(1)$. As the result, we have the following table.

\begin{tabular}{|c|c|}
\hline$\pi \cdot s_{p}$ & $s_{p} \cdot \tau$ \\
\hline$\overbrace{1 \rightarrow \pi(1)}^{\pi} \Rightarrow \overbrace{\pi(1) \rightarrow \pi(1)}^{s_{p}}$ & $\overbrace{1 \rightarrow 2}^{s_{p}} \Rightarrow \overbrace{2 \rightarrow \pi(1)}^{\tau}$ \\
\hline
\end{tabular}

Case $x=2$. Note, that $\tau(p)=p$. As the result, we have the following table.

\begin{tabular}{|c|c|}
\hline$\pi \cdot s_{p}$ & $s_{p} \cdot \tau$ \\
\hline$\overbrace{2 \rightarrow 2}^{\pi} \Rightarrow \overbrace{2 \rightarrow p}^{s_{p}}$ & $\overbrace{1 \rightarrow 2}^{s_{p}} \Rightarrow \overbrace{2 \rightarrow p}^{\tau}$ \\
\hline
\end{tabular}

Case $x=p$. Note, that there is the path of the point 1 over the decomposition of $\tau$, namely $1 \stackrel{1}{\rightarrow} 2 \stackrel{2}{\rightarrow} d_{1} \stackrel{2, m-2}{\longrightarrow} d_{m-1} \stackrel{m-2}{\longrightarrow} 1 \stackrel{m-1}{\longrightarrow} 2$, which means that $\tau(1)=2$.

As the result, we have the following table.

\begin{tabular}{|c|c|}
\hline$\pi \cdot s_{p}$ & $s_{p} \cdot \tau$ \\
\hline$\overbrace{p \rightarrow 1}^{\pi} \Rightarrow \overbrace{1 \rightarrow 2}^{s_{p}}$ & $\overbrace{p \rightarrow 1}^{s_{p}} \Rightarrow \overbrace{1 \rightarrow 2}^{\tau}$ \\
\hline
\end{tabular}


Case $x=d_{1}$. Note, that in this case $\tau\left(d_{1}\right)=\pi(p)=1$. As the result, we have the following table.

\begin{tabular}{|c|c|}
\hline$\pi \cdot s_{p}$ & $s_{p} \cdot \tau$ \\
\hline$\overbrace{d_{1} \rightarrow p}^{\pi} \Rightarrow \overbrace{p \rightarrow 1}^{s_{p}}$ & $\overbrace{d_{1} \rightarrow d_{1}}^{s_{p}} \Rightarrow \overbrace{d_{1} \rightarrow 1}^{\tau}$ \\
\hline
\end{tabular}

7) Let $d_{2}=p, d_{3}=p$ and $\pi(p)=1$. Note, that in this case $d_{2}, d_{3}$ are unique representations of $p$ in $D$. Construct $\tau$ as follows

$$
\tau=\left[d_{1}, d_{1}, d_{4}, d_{5}, \ldots, d_{m-1}, d_{m}\right]_{S}
$$

From the construction of $\tau$ it follows that for every point $x, x \notin\left\{1,2, p, d_{1}\right\}$, we have $\pi(x) \notin$ $\left\{1,2, p, d_{1}\right\}$. Therefore, $\left(\pi \cdot s_{p}\right)(x)=\pi(x)=\tau(x)=\left(s_{p} \cdot \tau\right)(x)$. Hence, it is enough to show that $\left(\pi \cdot s_{p}\right)(x)=\left(s_{p} \cdot \tau\right)(x)$ for every point $x$ from $\left\{1,2, p, d_{1}\right\}$.

Case $x=1$. Note, that $\tau(2)=\pi(1)$. As the result, we have the following table.

\begin{tabular}{|c|c|}
\hline$\overbrace{1 \rightarrow \pi(1)}^{\pi \cdot s_{p}} \Rightarrow \overbrace{\pi(1) \rightarrow \pi(1)}^{s_{p}}$ & $\overbrace{1 \rightarrow 2}^{s_{p} \cdot \tau} \Rightarrow \overbrace{2 \rightarrow \pi(1)}^{s_{p}}$ \\
\hline
\end{tabular}

Case $x=2$. Note, that $\tau(p)=p$. As the result, we have the following table.

\begin{tabular}{|c|c|}
\hline$\pi \cdot s_{p}$ & $s_{p} \cdot \tau$ \\
\hline$\overbrace{2 \rightarrow 2}^{\pi} \Rightarrow \overbrace{2 \rightarrow p}^{s_{p}}$ & $\overbrace{1 \rightarrow 2}^{s_{p}} \Rightarrow \overbrace{2 \rightarrow p}^{\tau}$ \\
\hline
\end{tabular}

Case $x=p$. Note, that there is the path of the point 1 over the decomposition of $\tau$, namely $1 \stackrel{1}{\rightarrow} 2 \stackrel{2}{\rightarrow} d_{1} \stackrel{2, m-2}{\longrightarrow} d_{m-1} \stackrel{m-2}{\longrightarrow} 1 \stackrel{m-1}{\longrightarrow} 2$, which means that $\tau(1)=2$. As the result, we have the following table.

\begin{tabular}{|c|c|}
\hline$\pi \cdot s_{p}$ & $s_{p} \cdot \tau$ \\
\hline$\overbrace{p \rightarrow 1}^{\pi} \Rightarrow \overbrace{1 \rightarrow 2}^{s_{p}}$ & $\overbrace{p \rightarrow 1}^{s_{p}} \Rightarrow \overbrace{1 \rightarrow 2}^{\tau}$ \\
\hline
\end{tabular}

Case $x=d_{1}$. Note, that in this case $\tau\left(d_{1}\right)=\pi(p)=1$. As the result, we have the following table.

\begin{tabular}{|c|c|}
\hline$\pi \cdot s_{p}$ & $s_{p} \cdot \tau$ \\
\hline$\overbrace{d_{1} \rightarrow p}^{\pi} \Rightarrow \overbrace{p \rightarrow 1}^{s_{p}}$ & $\overbrace{d_{1} \rightarrow d_{1}}^{s_{p}} \Rightarrow \overbrace{d_{1} \rightarrow 1}^{\tau}$ \\
\hline
\end{tabular}

The proof is complete.

Lemma 8. Let $\pi \in A$ be a permutation with decomposition $D=\left(d_{1}, \ldots, d_{m}\right), m \geq 3,2$ is fixed in $\pi$ over $D$ with trivial path. Then for every natural number $p, p \geq 3$, there exists $\tau \in A$ such that $\pi \cdot s_{p}=s_{p} \cdot \tau$ and $\tau$ has a decomposition $\left(t_{1}, \ldots, t_{l}\right)$, where $l \leq m$ and $p \notin\left\{t_{1}, \ldots, t_{l}\right\}$.

Proof. Note that the case, when there is no $k \in \overline{1, m}$ such that $d_{k}=p$, is already proved in Lemma 6; the case, when $\pi\left(d_{1}\right)=d_{3}$ with no $k \in \overline{4, m}$ such that $d_{3}=d_{k}$, is already proved in Lemma 7.

The proof is by induction on the decomposition length $m$. 
The basis. Let $d=\pi\left(d_{1}\right)$. Note that due to assumptions of the lemma, the permutation $\pi$ requires: 2 positions for $d_{1}, d_{m-1}$; at least 2 positions for non-trivial path of $d_{1} ; 1$ point for element $d_{2}$. The last means that $m \geq 5$.

We will prove basis for cases $m=5,6$.

1) If $m=5$, then $d=1$ and there is the following decomposition of $\pi$, namely $\pi=\left[d_{1}, d_{2}, d_{3}, d_{4}, d_{5}\right]_{S}$, where $d_{1}=d_{4}, d_{3}=d_{5}$ and $d_{2} \neq d_{1}, d_{2} \neq d_{3}$. Based on Lemma 6 it is enough to consider three cases depending on $p$ from $\left\{d_{1}, d_{2}, d_{3}\right\}$.

Case $p=d_{1}$. Let $\tau=\left[d_{2}\right]_{S}$. From direct calculation it follows that $\pi \cdot s_{d_{1}}=s_{d_{1}} \cdot \tau$.

Case $p=d_{2}$. Let $\tau=\left[d_{1}, d_{1}\right]_{s}$. From direct calculation it follows that $\pi \cdot s_{d_{2}}=s_{d_{2}} \cdot \tau$.

Case $p=d_{3}$. Let $\tau=\left[d_{2}, d_{1}, d_{1}\right]_{S}$. From direct calculation it follows that $\pi \cdot s_{d_{3}}=s_{d_{3}} \cdot \tau$.

2) If $m=6$, then $d$ is equal to 1 or is not. We consider cases: $d=1$ and $d \neq 1$ separately.

Let $d=1$. Then there is the next decomposition of $\pi$, namely $\pi=\left[d_{1}, d_{2}, d_{3}, d_{4}, d_{5}, d_{6}\right]_{S}$, where $d_{1}=d_{5}, d_{3}=d_{6}$ and $d_{2}, d_{4}$ are different from $d_{1}, d_{3}$.

Let $d_{2} \neq d_{4}$. Based on Lemma 6 it is enough to consider four cases depending on $p$ from $\left\{d_{1}, d_{2}, d_{3}, d_{4}\right\}$. In case $p=d_{1}$ let $\tau=\left[d_{2}, d_{4}, d_{4}, d_{3}\right]_{S}$. Direct calculation implies $\pi \cdot s_{d_{1}}=s_{d_{1}} \cdot \tau$. In case $p=d_{2}$ let $\tau=\left[d_{3}, d_{4}, d_{1}, d_{3}\right]_{s}$. Direct calculation implies $\pi \cdot s_{d_{2}}=s_{d_{2}} \cdot \tau$. In case $p=d_{3}$ let $\tau=\left[d_{2}, d_{1}, d_{4}\right]_{s}$. Direct calculation implies $\pi \cdot s_{d_{3}}=s_{d_{3}} \cdot \tau$. In case $p=d_{4}$ let $\tau=\left[d_{2}, d_{3}, d_{1}, d_{1}\right]_{S}$. Direct calculation implies $\pi \cdot s_{d_{4}}=s_{d_{4}} \cdot \tau$.

Let $d_{2}=d_{4}$. Note that $\pi\left(d_{2}\right)=d_{2}$. Based on Lemma 6 it is enough to consider two cases depending on $p$ from $\left\{d_{1}, d_{3}\right\}$. In case $p=d_{1}$ let $\tau=\left[d_{3}\right]_{s}$. From direct calculation it follows that $\pi \cdot s_{d_{1}}=s_{d_{1}} \cdot \tau$. In case $p=d_{3}$ let $\tau=\left[d_{1}, d_{1}\right]_{S}$. From direct calculation it follows that $\pi \cdot s_{d_{3}}=s_{d_{3}} \cdot \tau$.

Let $d \neq 1$. Then there is the next decomposition of $\pi$, namely $\pi=\left[d_{1}, d_{2}, d_{3}, d_{4}, d_{5}, d_{6}\right]_{S}$, where $d_{1}=d_{5}, d_{3}=d_{4}$ and points $d_{1}, d_{2}, d_{3}, d_{6}$ are pairwise different. Based on Proposition 1 we obtain $\pi=\left[d_{1}, d_{2}, d_{3}, d_{3}, d_{1}, d_{6}\right]_{S}=\left[d_{1}, d_{2}, d_{3}, d_{3}, d_{6}, d_{6}, d_{1}, d_{1}\right]_{S}=\left[d_{1}, d_{2}, d_{6}, d_{3}, d_{1}, d_{1}\right]_{s}$. Statement is directly implied from Lemma 7.

Induction step: case $m$ under assumption that for $l<m$ the statement holds. Let $d=\pi\left(d_{1}\right)$. Consider cases of $d$.

Let $d=1$. Consider different cases: $d_{1}=d_{m-1}=p ; d_{1} \neq p, d_{m-1} \neq p$ and $\pi^{-1}(p)=1$; $d_{1} \neq p, d_{m-1} \neq p$ and $\pi^{-1}(p) \neq 1$.

1) Let $d_{1}=d_{m-1}=p$. Then there are possible the next options.

i) Let $d_{m}=p$. From Proposition 1 we have

$\pi \cdot s_{p}=\left[p, d_{2}, \ldots, d_{m-2}, p, p\right]_{S} \cdot[p]_{S}=[p]_{S} \cdot\left[d_{2}, \ldots, d_{m-2}, p, p, p\right]_{S}=[p]_{S} \cdot\left[d_{2}, \ldots, d_{m-2}\right]_{S}=s_{p} \cdot \tau$, where $\tau=\left[d_{2}, \ldots, d_{m-2}\right]_{S}$.

ii) Let $d_{m} \neq p$. From Proposition 1 we have

$$
\begin{aligned}
\pi \cdot s_{p}=\left[p, d_{2}, \ldots, d_{m-2}, p, d_{m}\right]_{S} \cdot[p]_{S} & =[p]_{S} \cdot\left[d_{2}, \ldots, d_{m-2}, d_{m}, d_{m}, p, p, p\right]_{S} \\
& =[p]_{S} \cdot\left[d_{2}, \ldots, d_{m-2}, d_{m}, d_{m}\right]_{S}=s_{p} \cdot \tau,
\end{aligned}
$$

where $\tau=\left[d_{2}, \ldots, d_{m-2}, d_{m}, d_{m}\right]_{S}$.

2) Let $d_{1} \neq p, d_{m-1} \neq p$ and $\pi^{-1}(p)=1$. Then there are possible the next options.

i) Let $d_{2}=p$ and there is no $k \in \overline{3, m}$ such that $p=d_{k}$. Construct $\tau$ as follows $\tau=\left[d_{3}, \ldots, d_{m}\right]_{S}$. 
From the construction of $\tau$ it follows that for every point $x, x \notin\left\{1,2, p, d_{1}\right\}$, we have $\pi(x) \notin$ $\left\{1,2, p, d_{1}\right\}$. Therefore, $\left(\pi \cdot s_{p}\right)(x)=\pi(x)=\tau(x)=\left(s_{p} \cdot \tau\right)(x)$. Hence, it is enough to show that $\left(\pi \cdot s_{p}\right)(x)=\left(s_{p} \cdot \tau\right)(x)$ for every point $x$ from $\left\{1,2, p, d_{1}\right\}$.

Case $x=1$. Note, that $\pi(1)=\tau(2)$. As the result, we have the following table.

\begin{tabular}{|c|c|}
\hline$\pi \cdot s_{p}$ & $s_{p} \cdot \tau$ \\
\hline$\overbrace{1 \rightarrow p}^{\pi} \Rightarrow \overbrace{p \rightarrow 1}^{s_{p}}$ & $\overbrace{1 \rightarrow 2}^{s_{p}} \Rightarrow \overbrace{2 \rightarrow 1}^{\tau}$ \\
\hline
\end{tabular}

Case $x=2$. Note, that $\tau(p)=p$, because there are no $p$ in the decomposition of $\tau$. As the result, we have the following table.

\begin{tabular}{|c|c|}
\hline$\pi \cdot s_{p}$ & $s_{p} \cdot \tau$ \\
\hline$\overbrace{2 \rightarrow 2}^{\pi} \Rightarrow \overbrace{2 \rightarrow p}^{s_{p}}$ & $\overbrace{2 \rightarrow p}^{s_{p}} \Rightarrow \overbrace{p \rightarrow p}^{\tau}$ \\
\hline
\end{tabular}

Case $x=p$. From $d_{2}=p$ it follows that $\pi(p)=\tau(1)$. As the result, we have the following table.

\begin{tabular}{|c|c|}
\hline$\pi \cdot s_{p}$ & $s_{p \rightarrow \pi(p)} \cdot \tau$ \\
\hline$\overbrace{p \rightarrow \pi(p) \rightarrow \pi(p)}^{s_{p}}$ & $\overbrace{p \rightarrow 1}^{s_{p}} \Rightarrow \overbrace{1 \rightarrow \pi(p)}^{\tau}$ \\
\hline
\end{tabular}

Case $x=d_{1}$. Note, that $d_{1}$ has the unique representation in the decomposition of $\tau$ on the $(m-1)$ th position. It follows that there is the path of $d_{1}$ over the decomposition of $\tau$, namely $d_{1} \stackrel{m-1}{\rightarrow} 1 \stackrel{m}{\rightarrow} 2$. As the result, we have the following table.

\begin{tabular}{|c|c|}
\hline$\pi \cdot s_{p}$ & $s_{p} \cdot \tau$ \\
\hline$\overbrace{d_{1} \rightarrow 1}^{\pi} \Rightarrow \overbrace{1 \rightarrow 2}^{s_{p}}$ & $\overbrace{d_{1} \rightarrow d_{1}}^{s_{p}} \Rightarrow \overbrace{d_{1} \rightarrow 2}^{\tau}$ \\
\hline
\end{tabular}

ii) Let there is the non-trivial path of the point 1 over $D$ such that

$$
1 \stackrel{1}{\rightarrow} 2 \stackrel{2}{\rightarrow} d_{2} \stackrel{2, t_{1}}{\longrightarrow} \ldots \stackrel{t_{2}, x-2}{\longrightarrow} d_{x-2} \stackrel{x-2}{\longrightarrow} 1 \stackrel{x-1}{\longrightarrow} 2 \stackrel{x}{\rightarrow} p,
$$

where $x$ is the last position of $p$ in $D$ and $t_{1}, t_{2}$ are some natural numbers such that $t_{2}>t_{1}$.

Denote $\left[d_{t_{1}}, \ldots, d_{x-1}\right]_{s}$ by $\sigma$ and note that $\sigma$ satisfies conditions of the lemma. From the induction assumption it follows that there exists the decomposition of some permutation $\mu$ with the length less or equal $x-t_{1}$ such that $\sigma \cdot s_{p}=s_{p} \cdot \mu$.

As the result, for the permutation $\pi$ with the decomposition, which is transformed in corresponding way, the path of 1 will be trivial: $1 \stackrel{1}{\rightarrow} 2 \stackrel{2}{\rightarrow} p$. This case is already considered.

3) Let $d_{1} \neq p, d_{m-1} \neq p, \pi^{-1}(p) \neq 1$. Denote $\pi^{-1}(p)$ by $d$. Similar to the previous case, if $\operatorname{Tr}\left(d_{1}\right) \neq(1,3, m)$, then there exists the transformation of the decomposition $D$ into another decomposition $Y$, which has length less or equal $m$. And in this decomposition the equality $\operatorname{Tr}\left(d_{1}, \pi, Y\right)=(1,3, m)$ holds. Without loosing the generality, we can assume that $\operatorname{Tr}\left(d_{1}, \pi, D\right)=(1,3, m)$.

Consider different cases.

i) Let $d_{2}=p$. Construct $\tau$ as follows

$$
\tau=\left[t_{3}, \ldots, t_{m}\right]_{S}, \quad \text { where } t_{i}= \begin{cases}d_{m}, & \text { if } d_{i}=p, \\ d_{i}, & \text { otherwise }\end{cases}
$$


From the construction of $\tau$ it follows that for every point $x, x \notin\left\{1,2, p, d_{1}, d\right\}$, we have $\pi(x) \notin\left\{1,2, p, d_{1}, d\right\}$. Therefore, $\left(\pi \cdot s_{p}\right)(x)=\pi(x)=\tau(x)=\left(s_{p} \cdot \tau\right)(x)$. Hence, it is enough to show that $\left(\pi \cdot s_{p}\right)(x)=\left(s_{p} \cdot \tau\right)(x)$ for every point $x$ from $\left\{1,2, p, d_{1}, d\right\}$.

Case $x=1$. Note, that there is the next path of the point 1 in $D$, namely $1 \stackrel{1}{\rightarrow} 2 \stackrel{2}{\rightarrow} d_{2} \stackrel{2, x}{\Longrightarrow}$ $d_{x} \rightarrow \ldots \rightarrow \pi(1)$, where $x$ is the first position of $p$ in $D$ after 2 . In the decomposition of $\tau$ we have the next path of the point 2 , namely $2 \stackrel{1}{\rightarrow} d_{3} \stackrel{1, x-1}{\longrightarrow} t_{x} \rightarrow \ldots \rightarrow \pi(1)$, because of the substitution $p$ into $d_{m}=d_{3}$. As the result, we have the following table.

\begin{tabular}{|c|c|}
\hline$\pi \cdot s_{p}$ & $s_{p} \cdot \tau$ \\
\hline$\overbrace{1 \rightarrow \pi(1)}^{\pi} \Rightarrow \overbrace{\pi(1) \rightarrow \pi(1)}^{s_{p}}$ & $\overbrace{1 \rightarrow 2}^{s_{p}} \Rightarrow \overbrace{2 \rightarrow \pi(1)}^{\tau}$ \\
\hline
\end{tabular}

Case $x=2$. Note, that $\tau(p)=p$, because there are no $p$ in the decomposition of $\tau$. As the result, we have the following table.

\begin{tabular}{|c|c|}
\hline$\pi \cdot s_{p}$ & $s_{p} \cdot \tau$ \\
\hline$\overbrace{2 \rightarrow 2}^{\pi} \Rightarrow \overbrace{2 \rightarrow p}^{s_{p}}$ & $\overbrace{2 \rightarrow p}^{s_{p}} \Rightarrow \overbrace{p \rightarrow p}^{\tau}$ \\
\hline
\end{tabular}
table.

Case $x=p$. From $d_{2}=p$ it follows that $\pi(p)=\tau(1)$. As the result, we have the following

$$
\begin{array}{|c|c|}
\hline \overbrace{p \rightarrow \pi(p)}^{\pi \cdot s_{p}} \Rightarrow \overbrace{\pi(p) \rightarrow \pi(p)}^{s_{p}} & \overbrace{p \rightarrow 1}^{s_{p} \cdot \tau} \Rightarrow \overbrace{1 \rightarrow \pi(p)}^{s_{p}} \\
\hline
\end{array}
$$

Case $x=d_{1}$. Note, that $d_{1}$ has the unique representation in the decomposition of $\tau$ on the $(m-1)$ th position. From this it follows that there is the path of $d_{1}$ over the decomposition of $\tau$, namely $d_{1} \stackrel{m-1}{\longrightarrow} 1 \stackrel{m}{\rightarrow} 2$. As the result, we have the following table.

$$
\begin{array}{|c|c|}
\hline \pi \cdot s_{p} & s_{p} \cdot \tau \\
\hline \overbrace{d_{1} \rightarrow 1}^{\pi} \Rightarrow \overbrace{1 \rightarrow 2}^{s_{p}} & \overbrace{d_{1} \rightarrow d_{1}}^{s_{p}} \Rightarrow \overbrace{d_{1} \rightarrow 2}^{\tau} \\
\hline
\end{array}
$$

Case $x=d$. Note, that there is the next path of $d$ in $\pi: d \rightarrow \ldots \stackrel{x}{\rightarrow} d_{x}=p$, where $x$ is the last position of $p$ in $D$. In case $\tau$, based on substitution $p$ by $d_{m}$, we have the next path: $d \rightarrow \ldots \stackrel{x}{\rightarrow} d_{m} \stackrel{x, m}{\longrightarrow} d_{m} \stackrel{m}{\rightarrow} 1$. As the result, we have the following table.

$$
\begin{array}{|c|c|}
\hline \pi \cdot s_{p} & s_{p} \cdot \tau \\
\hline \overbrace{d \rightarrow p}^{\pi} \Rightarrow \overbrace{p \rightarrow 1}^{s_{p}} & \overbrace{d \rightarrow d}^{s_{p}} \Rightarrow \overbrace{d \rightarrow 1}^{\tau} \\
\hline
\end{array}
$$

ii) Let $d_{2} \neq p$. Construct $\tau$ as follows

$$
\tau=\left[t_{2}, t_{3}, \ldots, t_{m}\right]_{S}, \quad \text { where } t_{i}= \begin{cases}d_{m}, & \text { if } d_{i}=p, \\ d_{i}, & \text { otherwise }\end{cases}
$$

From the construction of $\tau$ it follows that for every point $x, x \notin\left\{1,2, p, d_{1}, d\right\}$, we have $\pi(x) \notin\left\{1,2, p, d_{1}, d\right\}$. Therefore, $\left(\pi \cdot s_{p}\right)(x)=\pi(x)=\tau(x)=\left(s_{p} \cdot \tau\right)(x)$. Hence, it is enough to show that $\left(\pi \cdot s_{p}\right)(x)=\left(s_{p} \cdot \tau\right)(x)$ for every point $x$ from $\left\{1,2, p, d_{1}, d\right\}$. 
Case $x=1$. Note, that $\pi(1)=\tau(2)$. As the result, we have the following table.

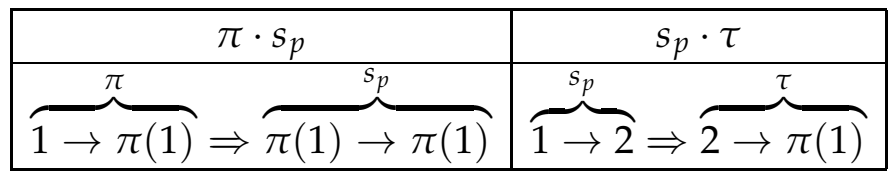

Case $x=2$. Note, that $\tau(p)=p$, because there are no $p$ in the decomposition of $\tau$. As the result, we have the following table.

\begin{tabular}{|c|c|}
\hline$\pi \cdot s_{p}$ & $s_{p} \cdot \tau$ \\
\hline$\overbrace{2 \rightarrow 2}^{\pi} \Rightarrow \overbrace{2 \rightarrow p}^{s_{p}}$ & $\overbrace{2 \rightarrow p}^{s_{p}} \Rightarrow \overbrace{p \rightarrow p}^{\tau}$ \\
\hline
\end{tabular}

Case $x=p$. Note, that in the decomposition of $\tau$, the path of the point 1 is the following $1 \stackrel{1}{\rightarrow} 2 \stackrel{2}{\rightarrow} t_{3} \stackrel{3, x}{\Longrightarrow} t_{x} \rightarrow \ldots \rightarrow \pi(p)$, where $x$ is the first position of $p$ in the decomposition $D$. As the result, we have the following table.

$$
\begin{array}{|c|c|}
\hline \overbrace{p \rightarrow \pi(p)}^{\pi \cdot s_{p}} \Rightarrow \overbrace{\pi(p) \rightarrow \pi(p)}^{s_{p}} & \overbrace{p \rightarrow 1}^{s_{p} \cdot \tau} \Rightarrow \overbrace{1 \rightarrow \pi(p)}^{s_{p}} \\
\hline
\end{array}
$$

Case $x=d_{1}$. Note, that $d_{1}$ has the unique representation in the decomposition of $\tau$ on the $(m-1)$ th position. It follows that there is the path of $d_{1}$ over the decomposition of $\tau$, namely $d_{1} \stackrel{m-1}{\rightarrow} 1 \stackrel{m}{\rightarrow} 2$. As the result, we have the following table.

\begin{tabular}{|c|c|}
\hline$\pi \cdot s_{p}$ & $s_{p} \cdot \tau$ \\
\hline$\overbrace{d_{1} \rightarrow 1}^{\pi} \Rightarrow \overbrace{1 \rightarrow 2}^{s_{p}}$ & $\overbrace{d_{1} \rightarrow d_{1}}^{s_{p}} \Rightarrow \overbrace{d_{1} \rightarrow 2}^{\tau}$ \\
\hline
\end{tabular}

Case $x=d$. Note, that we have the next path of $d$ in $\pi$, namely $d \rightarrow \ldots \stackrel{x}{\rightarrow} d_{x}=p$, where $x$ is the last position of $p$ in $D$. In case $\tau$, based on the substitution $p$ by $d_{m}$, we have the next path of $d$, namely $d \rightarrow \ldots \stackrel{x}{\rightarrow} d_{m} \stackrel{x, m}{\longrightarrow} d_{m} \stackrel{m}{\rightarrow} 1$. As the result, we have the following table.

\begin{tabular}{|c|c|}
\hline$\pi \cdot s_{p}$ & $s_{p} \cdot \tau$ \\
\hline$\overbrace{d \rightarrow p}^{\pi} \Rightarrow \overbrace{p \rightarrow 1}^{s_{p}}$ & $\overbrace{d \rightarrow d}^{s_{p}} \Rightarrow \overbrace{d \rightarrow 1}^{\tau}$ \\
\hline
\end{tabular}

Let $d \neq 1$. Then there exist natural numbers $x, y$ and the path of $d=d_{y}$ in $\pi$ over $D$ such that

$$
d_{1} \stackrel{1}{\rightarrow} 1 \stackrel{2}{\rightarrow} 2 \stackrel{3}{\rightarrow} d_{3} \Rightarrow \ldots \stackrel{x, y-2}{\longrightarrow} d_{y-2} \stackrel{y-2}{\longrightarrow} 1 \stackrel{y-1}{\longrightarrow} 2 \stackrel{y}{\rightarrow} d_{y},
$$

where $y$ is the last position of $d$ in $D$.

The above means that $\operatorname{Tr}\left(d_{1}, \pi, D\right)=\left(t_{1}, r_{1}, \ldots, t_{u}, r_{u}, y\right)$ for some natural numbers $t_{1}, r_{1}, \ldots, t_{u}, r_{u} \in \overline{3, y-2}, u \geq 0$. Let $\pi_{k}=\left[d_{t_{k}}, \ldots, d_{r_{k+1}}, d_{r_{k+1}+1}\right]$ for every $k \in \overline{1, u}$. Note, that $\pi_{k}$ satisfies assumptions of the lemma and the length of its decomposition is less than $m$. So, based on the induction assumption, for every natural $p, p \geq 3$, we have $\pi_{k} \cdot s_{p}=s_{p} \cdot \tau_{k}$ for some $\tau_{k}$ with the length of the decomposition less or equal to $r_{k}-t_{k}+2$. Let fix $\tau_{k}$ for case $p=d$. Let $v=\left[d_{1}, d_{2}\right]_{S}$ and $\mu=\left[d_{y+1}, \ldots, d_{m}\right]_{S}$. Then

$$
\pi=v \cdot \pi_{1} \cdot \ldots \cdot \pi_{u} \cdot s_{d} \cdot \mu=v \cdot \pi_{1} \cdot \ldots \cdot \pi_{u-1} \cdot s_{d} \cdot \tau_{u} \cdot \mu=\ldots=v \cdot s_{d} \cdot \tau_{1} \cdot \ldots \cdot \tau_{u} \cdot \mu .
$$


Note, that the transformation from $\pi_{k}$ to $\tau_{k}$ does not introduce points $d_{1}, d_{m-1}$, because they are unique represented in $D$ and they are not represented in the decomposition of $\pi_{k}$, $k \in \overline{1, u}$; there are no $d$ in the decomposition $Y$ of $\pi$, which is the concatenation of considered decompositions $v,[d]_{s}, \tau_{1}, \ldots, \tau_{u}, \mu, \pi\left(d_{1}\right)=d$ on the 3rd position in $Y$.

Applying Lemma 7 the statement follows.

\section{Stability and strong growing}

\subsection{Stability of $\mathbb{A}$}

Proposition 3. Let $\pi$ be a permutation from $A, D=\left(d_{1}, \ldots, d_{m}\right)$ be its decomposition over $S, p$ be a point from $\left\{d_{1}, \ldots, d_{m}\right\}, \pi(p) \neq 1,2$ and $\operatorname{Tr}(p, \pi, D)=\left(x, t_{1}, r_{1}, \ldots, t_{u}, r_{u}, y\right)$ for some natural $u$, where $x$ is the first position of $p$ in $D, y$ is the last position of $\pi(p)$ in $D$, $t_{1}, r_{1}, \ldots, t_{u}, r_{u} \in \overline{x+2, y-2}$. Then $\pi$ has a decomposition

$$
Y=\left(d_{1}, \ldots, d_{x-1}, q_{1}, \ldots, q_{z-1}, p, d_{y-1}, d_{y}, \ldots, d_{m}\right)
$$

of length $\leq m$ such that $p$ has trivial path in $\pi$ over $Y$, i.e. $\operatorname{Tr}(p, \pi, Y)=(v-2, v)$, where $v$ is the last position of $d_{y}$ in $Y$.

Proof. Set $\pi_{k}=\left[d_{t_{k}-1}, d_{t_{k}}, \ldots, d_{r_{k}}\right]_{S}, k \in \overline{1, u}$. Lemma 5 implies that $s_{p} \cdot \pi_{k}=\tau_{k} \cdot s_{p}$ for some $\tau_{k}$ with a decomposition, whose length is less or equal to $r_{k}-t_{k}+2$ and that does not contain $p$.

Let $v=\left[d_{1}, \ldots, d_{x-1}\right]_{S}$ and $\mu=\left[d_{y-1}, \ldots, d_{m}\right]_{S}$. Then

$$
\pi=v \cdot s_{p} \cdot \pi_{1} \cdot \ldots \pi_{u} \cdot \mu=\ldots=v \cdot \tau_{1} \cdot \ldots \cdot \tau_{u} \cdot s_{p} \cdot \mu .
$$

Let $Y$ be a decomposition of $\pi$, obtained as the concatenation of decompositions of permutations $v, \tau_{1}, \ldots, \tau_{u}, s_{p}, \mu$. Then the length of the decomposition $Y$ is not greater than $m$ and $p$ has trivial path in $\pi$ over $Y$. The proof is complete.

Corollary 1. Let $\pi$ be a permutation from $A, D=\left(d_{1}, \ldots, d_{m}\right)$ be its decomposition over $S$, $p$ be a point from $\left\{d_{1}, \ldots, d_{m}\right\}, \pi(p) \in\{1,2\}$ and $\operatorname{Tr}(p, \pi, D)=\left(x, t_{1}, r_{1}, \ldots, t_{u}, r_{u}\right)$ for some natural $u$, where $x$ is the first position of $p$ in $D, t_{1}, r_{1}, \ldots, t_{u}, r_{u} \in \overline{x+2, m-2}$. Then $\pi$ has a decomposition

$$
Y= \begin{cases}\left(d_{1}, \ldots, d_{x-1}, q_{1}, \ldots, q_{z-1}, p\right), & \text { if } \pi(p)=1, \\ \left(d_{1}, \ldots, d_{x-1}, q_{1}, \ldots, q_{z-1}, p, d_{m}\right), & \text { if } \pi(p)=2\end{cases}
$$

of length $\leq m$ such that $p$ has trivial path in $\pi$ over $Y$, i.e. $\operatorname{Tr}(p, \pi, Y)=(v)$, where $v=$ $x+z-1$.

Proof. The statement directly follows from Proposition 3.

Proposition 4. Let $\pi$ be a permutation from $A, D=\left(d_{1}, \ldots, d_{m}\right)$ be its decomposition over $S$ such that there exists a point $p \in\left\{d_{1}, \ldots, d_{m}\right\}$, not contained in $\sup p(\pi)$. Then the decomposition $D$ can be transformed to another decomposition of $\pi$ with the length, which is shorter at least by 1 .

Proof. Note that $p \notin \sup p(\pi)$ implies that $\pi(p)=p \neq 1,2$. Using Proposition 3, we can assume that the point $p$ has trivial path in $\pi$ over $D$. Hence, there exists $y \in \overline{1, m-2}$ such that $\operatorname{Tr}(p, \pi, D)=(y-2, y)$. 
Consider different cases depending on the value of $d_{y-1}$.

1) If $d_{y-1}=p$, then $s_{p} \cdot d_{y-1} \cdot s_{p}=e$. As the result, we have

$$
\pi=\left[d_{1}, \ldots, d_{y-3}, p, p, p, d_{y+1}, \ldots, d_{m}\right]_{S}=\left[d_{1}, \ldots, d_{y-3}, d_{y+1}, \ldots, d_{m}\right]_{S} .
$$

Hence, $Y=\left(d_{1}, \ldots, d_{y-3}, d_{y+1}, \ldots, d_{m}\right)$ is the decomposition of $\pi$ with the length less than $m$ by 3 and $p \notin\left\{d_{1}, \ldots, d_{y-3}, d_{y+1}, \ldots, d_{m}\right\}$.

2) If $d_{y-1} \neq p$, then $s_{p} \cdot d_{y-1} \cdot s_{p}=d_{y-1} \cdot d_{y-1}$, and

$$
\pi=\left[d_{1}, \ldots, d_{y-3}, p, d_{y-1}, p, d_{y+1}, \ldots, d_{m}\right]_{S}=\left[d_{1}, \ldots, d_{y-3}, d_{y-1}, d_{y-1}, d_{y+1}, \ldots, d_{m}\right]_{s} .
$$

Hence, $Y=\left(d_{1}, \ldots, d_{y-3}, d_{y-1}, d_{y-1}, d_{y+1}, \ldots, d_{m}\right)$ is the decomposition of $\pi$ with the length less than $m$ by 1 and $p \notin\left\{d_{1}, \ldots, d_{y-1}, d_{y+3}, \ldots, d_{m}\right\}$.

Proposition 5. Let $\pi$ be a permutation from $A$ and $D$ be its decomposition over $S, p$ be a point from $D, p \notin \sup p(\pi)$ and the last two occurrences of $p$ in $D$ are beside. Then the decomposition $D$ can be transformed to another decomposition of $\pi$ with the length, which is shorter at least by 3 .

Proof. It follows from proof of Proposition 4 with $d_{y-1}=p$.

Proposition 6. Let $\pi$ be a permutation from $A, D=\left(d_{1}, \ldots, d_{m}\right)$ be its decomposition over $S, p$ be a point from $\left\{d_{1}, \ldots, d_{m}\right\}, \pi(p) \neq 1,2$ and $\operatorname{Tr}(p, \pi, D)=\left(x, t_{1}, r_{1}, \ldots, t_{u}, r_{u}, y\right)$ for some natural $u$, where $x$ is the first position of $p$ in $D, y$ is the last position of $\pi(p)$ in $D$, $t_{1}, r_{1}, \ldots, t_{u}, r_{u} \in \overline{x+2, y-2}$.

Then $\pi$ has decomposition

$$
Y=\left(d_{1}, \ldots, p, q_{1}, d_{y}, q_{3}, \ldots, q_{z}, d_{y+1}, \ldots, d_{m}\right)
$$

of length $\leq m$ such that $p$ has trivial path in $\pi$ over $Y$, i.e. $\operatorname{Tr}(p, \pi, Y)=(x, x+2)$.

Proof. Let $\pi_{k}=\left[d_{t_{k}}, \ldots, d_{r_{k}}, d_{r_{k}+1}\right]_{S}$ for every $k \in \overline{1, u}$ and $d=d_{y}$. From Lemma 8 it follows that $\pi_{k} \cdot s_{d}=s_{d} \cdot \tau_{k}$ for some $\tau_{k}$ with decomposition, whose length is less or equal to $r_{k}-t_{k}+2$ and that does not include $d$.

Let $v=\left[d_{1}, \ldots, d_{x+1}\right]_{S}$ and $\mu=\left[d_{y+1}, \ldots, d_{m}\right]_{S}$. Then

$$
\pi=v \cdot \pi_{1} \cdot \ldots \pi_{u} \cdot s_{d} \cdot \mu=\ldots=v \cdot s_{d} \cdot \tau_{1} \cdot \ldots \cdot \tau_{u} \cdot \mu .
$$

Then required decomposition $Y$ is the concatenation of decompositions $v, s_{d}, \tau_{1}, \ldots, \tau_{u}, \mu$. Its length is less or equal to the length of $D$. Finally, $\operatorname{Tr}(p, \pi, Y)=(x, x+2)$.

Corollary 2. Let $\pi$ be a permutation from $A, D=\left(d_{1}, \ldots, d_{m}\right)$ be its decomposition over $S$, $p \in\{1,2\}$ be a point, $\pi(p) \in\left\{d_{1}, \ldots, d_{m}\right\}$ and $\operatorname{Tr}(p, \pi, D)=\left(t_{1}, r_{1}, \ldots, t_{u}, r_{u}, y\right)$ for some natural $u$, where $y$ is the last position of $\pi(p)$ in $D, t_{1}, r_{1}, \ldots, t_{u}, r_{u} \in \overline{x+2, y-2}$. Then $\pi$ has decomposition

$$
Y= \begin{cases}\left(d_{1}, d_{y}, q_{1}, \ldots, q_{z}, d_{y+1}, \ldots, d_{m}\right), & \text { if } p=1, \\ \left(d_{y}, q_{1}, \ldots, q_{z}, d_{y+1}, \ldots, d_{m}\right), & \text { if } p=2\end{cases}
$$

of length $\leq m$ such that $p$ has trivial path in $\pi$ over $Y$, i.e. $\operatorname{Tr}(p, \pi, Y)=(1)$ or $\operatorname{Tr}(p, \pi, Y)=(2)$ based on $p=1,2$.

Proof. The statement directly follows from from Proposition 6. 
Proposition 7. Let $\pi$ be a permutation from $A$ and $D$ be its decomposition over $S, p$ be a point from $D, p \notin \operatorname{supp}(\pi)$ and the first two occurrences of $p$ in $D$ are beside. Then the decomposition $D$ can be transformed to another decomposition of $\pi$ with the length, which is shorter at least by 3 .

Proof. From $p \notin \sup p(\pi)$ it follows that $\pi(p)=p \neq 1$, 2. By Proposition 3 we can assume that there exists $x \in \overline{1, m-2}$ such that $\operatorname{Tr}(p, \pi, D)=(x, x+2)$. Then $d_{x+1}=p$. From Proposition 1 we have

$$
\left[d_{1}, \ldots, d_{x-1}, p, p, p, d_{x+3}, \ldots, d_{m}\right]_{S}=\left[d_{1}, \ldots, d_{x-1}, d_{x+3}, \ldots, d_{m}\right] .
$$

Hence, $Y=\left(d_{1}, \ldots, d_{x-1}, d_{x+3}, \ldots, d_{m}\right)$ is the decomposition of $\pi$, whose length is less than $m$ by 3 and $p \notin\left\{d_{1}, \ldots, d_{x-1}, d_{x+3}, \ldots, d_{m}\right\}$.

Theorem 1. The series $\mathbb{A}$ is stable.

Proof. Suppose that the series $\mathbb{A}$ is not stable. Then there are some natural numbers $N_{1}, N_{2}$, $N_{1}>N_{2}$, and there is a permutation $\pi \in A l t\left(N_{1}\right)$ such that $|a|_{S o G\left(N_{1}\right)}>|a|_{S o G\left(N_{2}\right)}=: m$.

Let $D$ be the minimal decomposition of $\pi$ over $\operatorname{SoG}\left(N_{2}\right)$. Note, that $D$ contains at least one generator from $\operatorname{SoG}\left(N_{2}\right) \backslash S o G\left(N_{1}\right)$. Otherwise, $\pi$ can be decomposed over $D$ in $A l t\left(N_{1}\right)$, with the length $\leq|a|_{S o G\left(N_{1}\right)}$. Then there is at least one point from $\overline{N_{1}+1, N_{2}}$, which is in $D$ and not in $\operatorname{supp}(\pi)$, as $\pi \in A l t\left(N_{1}\right)$. Then the decomposition $D$ can be transformed into shorter decomposition $T$ with length $l$ by removing all points $\left\{N_{1}+1, \ldots, N_{2}\right\} \cap D$ based on Proposition 4 . Note, that the decomposition $T$, obtained by this way, is over $\operatorname{SoG}\left(N_{1}\right)$. As the result, we have

$$
|a|_{S o G\left(N_{1}\right)}>|D|=m>l=|T| \geq|a|_{S o G\left(N_{1}\right)} .
$$

This leads to a contradiction, which complete the proof.

\subsection{Strong growing of systems of generators $\operatorname{SoG}(n), n \geq 3$}

Lemma 9. Let a be some element of Alt $n)$ with the minimal decomposition $D=\left(d_{1}, \ldots, d_{m}\right)$ over SoG $(n)$. Then for $a \cdot s_{n+1}$ the decomposition $Y=\left(d_{1}, \ldots, d_{m}, n+1\right)$ will be the minimal decomposition over $\operatorname{SoG}(n+1)$.

Proof. Suppose that there exists the minimal decomposition $Y=\left(y_{1}, \ldots, y_{l}\right)$ of $a \cdot s_{n+1}$ over SoG $(n+1)$ such that $l<m+1$. Then the decomposition $R=\left(y_{1}, \ldots, y_{l}, n+1, n+1\right)$ will be the decomposition of $a \cdot s_{n+1} \cdot s_{n+1} \cdot s_{n+1}=a$ over $\operatorname{SoG}(n+1)$. Note, that the decomposition $R$ can be transformed to the decomposition $W$, which length is shorter at least 3 to $R$ and without point $n+1$, according to Proposition 5. So, the decomposition $W$ will be the decomposition of $a$ over $\operatorname{SoG}(n)$ with length $\leq l+2-3=l-1$. As the result, we have the following inequality

$$
m+1=|a|_{S o G(n)}+1 \leq|W|+1 \leq l=\left|a \cdot s_{n+1}\right|_{S o G(n+1)}<m+1 .
$$

This contradiction completes the proof.

Lemma 10. Let a be an element from $A l t(n)$ with the minimal decomposition $D=\left(d_{1}, \ldots, d_{m}\right)$ over SoG $(n)$. Then for $s_{n+1} \cdot a$ the decomposition $Y=\left(n+1, d_{1}, \ldots, d_{m}\right)$ is the minimal decomposition over SoG $(n+1)$.

Proof. Proof is similar to Lemma 9 with using Proposition 7. 
Theorem 2. The system of generators $\operatorname{So} G(n)$ is strong growing for every natural number $n \geq 3$.

Proof. Let $\pi$ be a diameter element from $\operatorname{Alt}(n)$. Suppose that the element $\pi$ is not full generated. Then there is some point $p \in \overline{3, n}$ such that $p \notin \sup p(\pi)$. So, based on Lemma 9 we obtain the inequality

$$
\left|\pi \cdot s_{p}\right|=|a|_{S o G(n)}+1>|a|_{S o G(n)}=\operatorname{Diam}_{S}(\operatorname{Alt}(n)) .
$$

This leads to a contradiction, which complete the proof.

\section{Computation of diameters}

In this section we show that the groups-generators series $\mathbb{A}$ is $C$-constant, uniform and homogeneous. These properties were introduced in [8]. It gives us an opportunity to apply the homogeneous down search algorithm to obtain exact values for the diameter of $A l t(n)$ over $\operatorname{SoG}(n)$ for $n \leq 43$.

\subsection{Properties of groups-generators series}

Let $\mathbb{G}$ be a groups-generators series. Let $\operatorname{GDiff}(n)$ be the set of generators, which appear exactly on the $n$ th, $n \geq 1$, i.e.

$$
\operatorname{GDiff}(1)=\operatorname{SoG}(1), \quad \operatorname{GDiff}(n)=\operatorname{SoG}(n) \backslash \operatorname{SoG}(n-1), \quad n \geq 2 .
$$

Definition 4. The groups-generators series $\mathbb{G}$ is called uniform if

$$
\left\langle\bigcup_{k=1}^{t} G \operatorname{Diff}\left(i_{k}\right)\right\rangle \simeq G(t)
$$

for every index tuple $I=\left(i_{i}, i_{2}, \cdots, i_{t}\right)$ of cardinality $t$.

Let $C$ be a natural number.

Definition 5. The groups-generators series $\mathbb{G}$ is called C-stable if $|G \operatorname{Diff}(t)|=C, t \geq 1$.

Let the groups-generators series $\mathrm{G}$ be $C$-stable. Suppose that elements from $\bigcup_{n \geq 1} \operatorname{SoG}(n)$ are enumerated, i.e.

$$
\bigcup_{n \geq 1} \operatorname{SoG}(n)=\left\{s_{i} \in \mathbb{G} \mid i \in \mathbb{N}\right\}
$$

and the following conditions hold:

1) $\operatorname{SoG}(n)=\left\{s_{1}, s_{2}, \ldots, s_{C}, s_{C}+1, \ldots, s_{n \cdot C}\right\}, n \geq 1$,

2) $\operatorname{GDiff}(n)=\left\{s_{(n-1) \cdot C+1}, s_{(n-1)} \cdot C+2, \ldots, s_{n \cdot C}\right\}, n \geq 1$.

Let $I=\left(i_{1}, i_{2}, \cdots, i_{t}\right)$ be an index tuple. Define the mapping $h_{I}^{C}$ from $\overline{1, t \cdot C}$ to $\bigcup_{k=1}^{t} \overline{\left(i_{k}-1\right) \cdot C+1, i_{k} \cdot C}$ by the rule

$$
h_{I}^{C}(x)=\left(i_{[(x-1) / C]+1}-1\right) \cdot C+(x-1) \bmod C+1 .
$$


Note, that the unique representation of $x=(k-1) \cdot C+r, k \in \overline{1, n}, r \in \overline{1, C}$ leads to the equality

$$
h_{I}^{C}((k-1) \cdot C+r)=\left(i_{k}-1\right) \cdot C+r .
$$

The last equality can be reinterpreted as follows: if $x$ is the index of the $r$ th generator of $\operatorname{GDiff}(k)$, then $h_{I}^{C}(x)$ is the index of the $r$ th generator of $\operatorname{Giff}\left(i_{k}\right)$.

Now define the mapping $\psi_{I}^{C}: \operatorname{SoG}(n) \rightarrow \bigcup_{k=1}^{n} G \operatorname{Diff}\left(i_{k}\right)$ by the rule

$$
\psi_{I}^{C}\left(s_{i}\right)=s_{h_{I}^{C}(i)} \cdot
$$

We will use notations

1) $\operatorname{SoG}_{I}(n)=\bigcup_{k=1}^{n} \operatorname{GDiff}\left(i_{k}\right)$;

2) $G_{I}(n)=\left\langle\operatorname{SoG}_{I}(n)\right\rangle$.

Note, that $\operatorname{SoG}_{I}(n)$ is the image of $\operatorname{SoG}(n)$ under $\psi_{I}^{C}$.

Definition 6. A uniform and C-stable groups-generators series $\mathbb{G}$ is called homogeneous if for every natural $t$ and every index tuple $I$ of cardinality $t$ the mapping $\psi_{I}^{C}$ can be extended to the group isomorphism between $G(t)$ and $G_{I}(t)$.

We will omit the letter $C$ in notations $\psi_{I}^{C}, h_{I}^{C}$. In this paper, we will use notations $\psi_{I}, h_{I}$ instead, unless otherwise stated.

\subsection{Homogeneity of $\mathbb{A}$}

It is straightforward that the groups-generators series $\mathbb{A}$ is 1-constant and $\operatorname{GDiff}(n)=$ $\{(1,2, n)\}, n \geq 3$. We consider index tuples as tuples of natural numbers, which are greater of equal 3. Note, that in case of alternating groups-generators series $\mathbb{A}$ for every index tuple $I=\left(i_{3}, \ldots, i_{t}\right)$ we have $h_{I}^{C}(x)=i_{x-1+1}-1+1=i_{x}$.

So, $\psi_{I}^{1}: \operatorname{SoG}(n) \rightarrow \bigcup_{k=1}^{n} \operatorname{GDiff}\left(i_{k}\right) ; \psi_{I}^{1}\left(s_{k}\right)=s_{i_{k}}$ for every $k \in \overline{3, n}$.

We will use notation $\psi_{I}$ instead of $\psi_{I}^{1}$.

Theorem 3. The groups-generators series $\mathbb{A}$ is homogeneous.

Proof. Let $n$ be some natural number greater than 2 and $I=\left(i_{3}, \ldots, i_{n}\right)$ be some index tuple of cardinality $n-2$. For uniform property, it is enough to show that

$$
A:=\left\langle\bigcup_{k=3}^{n} \operatorname{GDiff}\left(i_{k}\right)\right\rangle \approx \operatorname{Alt}(n)
$$

Note, that for any natural $k \in \overline{3, n}$ we have $\operatorname{GDiff}\left(i_{k}\right)=\left\{\left(1,2, i_{k}\right)\right\}$. So,

$$
\left\langle\bigcup_{k=3}^{n} \operatorname{GDiff}\left(i_{k}\right)\right\rangle=\left\langle\bigcup_{k=3}^{n}\left\{\left(1,2, i_{k}\right)\right\}\right\rangle=\left\langle\left\{\left(1,2, i_{k}\right) \mid k \in \overline{3, n}\right\}\right\rangle .
$$

Consider the mapping $\psi_{I}: \operatorname{SoG}(n) \rightarrow S_{0} G_{I}(n)$ which maps the set $\{(1,2, k): k \in \overline{3, n}\}$ into $\left\{\left(1,2, i_{k}\right): k \in \overline{3, n}\right\}$ as follows $\psi_{I}((1,2, k))=\left(1,2, i_{k}\right)$. Note, that the mapping $\psi_{I}$, 
which is bijection from $\operatorname{SoG}(n)$ to $\operatorname{SoG}_{I}(n)$, is based on the bijection of natural numbers $\phi_{I}:\{1,2,3, \ldots, n\} \rightarrow\left\{1,2, i_{3}, \ldots, i_{n}\right\}$, defined by

$$
\phi_{I}(x)= \begin{cases}1, & \text { if } x=1, \\ 2, & \text { if } x=2, \\ i_{x}, & \text { if } x \in \overline{3, n}\end{cases}
$$

From the last it follows that $\psi_{I}$ is isomorphism over groups $G(n)$ and $G_{I}(n)$. Based on definition, the groups-generators series $\mathbb{A}$ is uniform. Moreover, the mapping $\psi_{I}$, which is defined from 1-constant property of $\mathbb{A}$, has been extended to the group isomorphism. Hence, the groups-generators series $\mathbb{A}$ is homogeneous.

\subsection{Application of homogeneous down search algorithm}

Theorem 3 implies that the homogeneous down search algorithm, which was introduced in [8], can be applied to the alternating group $A l t(n)$ and the system of generators $\operatorname{SoG}(n)$ for every natural number $n \geq 3$.

Homogeneous down search algorithm was implemented on computer algebra system SageMath. The solution was run with the system resources, stated in the following table.

\begin{tabular}{|c|c|c|c|c|}
\hline OS & cpu & memory & SageMath & Python \\
\hline Ubuntu 18.04 & i7-9750H & $16 \mathrm{gb}$ & 9.1 & 3.7 .3 \\
\hline
\end{tabular}

The results of the application of homogeneous down search to alternating groups $\operatorname{Alt}(n)$ with systems of generators $\operatorname{SoG}(n)=((1,2,3), \ldots,(1,2, n))$ for $n \in \overline{3,43}$ are summirazied in the following table, where $D(n)$ denotes the diameter of $A l t(n)$ over the system of generators SoG $(n)$.

\begin{tabular}{|c|c|c|c|c|c|c|c|c|c|}
\hline$n$ & $D(n)$ & $n$ & $D(n)$ & $n$ & $D(n)$ & $n$ & $D(n)$ & $n$ & $D(n)$ \\
\hline 4 & 4 & 12 & 16 & 20 & 28 & 28 & 40 & 36 & 52 \\
5 & 5 & 13 & 17 & 21 & 29 & 29 & 41 & 37 & 53 \\
6 & 6 & 14 & 18 & 22 & 30 & 30 & 42 & 38 & 54 \\
7 & 8 & 15 & 20 & 23 & 32 & 31 & 43 & 39 & 56 \\
8 & 10 & 16 & 22 & 24 & 34 & 32 & 44 & 40 & 58 \\
9 & 11 & 17 & 23 & 25 & 35 & 33 & 47 & 41 & 59 \\
10 & 12 & 18 & 24 & 26 & 36 & 34 & 48 & 42 & 60 \\
11 & 14 & 19 & 26 & 27 & 38 & 35 & 50 & 43 & 62 \\
\hline
\end{tabular}

\section{References}

[1] Akers Sh.B. (Jr), Krishnamurthy B., Harel D. The Star Graph: An Attractive Alternative to the n-Cube. In: Proc. of the Intern. Conf. on Parallel Processing, ICPP'87, University Park, PA, USA, August 1987, Pennsylvania State University Press, Pennsylvania, 1987, 393-400.

[2] Babai L., Seress A. On the diameter of permutation groups. European J. Combin. 1992, 13 (4), $231-243$. doi:10.1016/S0195-6698(05)80029-0

[3] Bajpai J., Dona D., Helfgott H.A. Growth estimates and diameter bounds for classical Chevalley groups. 2021, arXiv:2110.02942v1. 
[4] Breuillard E., Green B., Tao T. Approximate subgroups of linear groups. Geom. Funct. Anal. 2011, 21, article number 774. doi:10.1007/s00039-011-0122-y

[5] Even S., Goldreich O. The minimum-length generator sequence problem is NP-hard. J. Algorithms 1981, 2 (3), 311313. doi:10.1016/0196-6774(81)90029-8

[6] Helfgott H.A. Growth and generation in $S L_{2}(\mathbb{Z} / p \mathbb{Z})$. Ann. of Math. (2) 2008, 167 (2), 601-623. doi:10.4007/annals.2008.167.601

[7] Helfgott H.A., Seress A. On the diameter of permutation groups. Ann. Math. (2) 2014,179 (2), $611-658$. doi:10.4007/annals.2014.179.2.4

[8] Olshevskyi M. Diameter search algorithms for directed Cayley graphs. Mohyla Math. J. 2021, 4 (in press).

[9] Pyber L., Szabó E. Growth in finite simple groups of Lie type of bounded rank. 2011, arXiv:1005.1858v2.

Received 04.09.2021

Ольшевський М.С. Метричні властивості графів Келі знакозмінних груп // Карпатські матем. публ. - 2021. - Т.13, №2. - С. 545-581.

Аана робота розглядається в контексті розв'язку добре відомої задачі пошуку діаметру скінченної групи по заданій системі твірних. На основі заданої групи та ії системи твірних будується граф Келі. Аля цього графа знаходиться діаметр, що називається діаметром групи відносно системи твірних.

Розглядаються групи парних підстановок з класичною незвідною системою твірних, шо складається з циклів довжини три виду $(1,2, k)$. В роботі проводиться аналіз властивостей розкладів парних підстановок відносно даної системи твірних і послідовності знакозмінних груп з вказаними системами твірних. Виводиться певне правило пересування твірного елемента в розкладі підстановки, окремо для руху зліва направо та справо наліво. Таким чином введене правило дозволяє прибирати з розкладу ті твірні елементи, шо визначають нерухомі точки підстановки. Ааний результат дає можливість довести, що система твірних зберігає мінімальність розкладів елементів при зростанні. Як наслідок, показано, шо система твірних $є$ строго зростаючою системою твірних.

В роботі використовується теорія однорідності, введена у попередній роботі автора. $\Delta л я$ послідовності груп парних підстановок з вказаними системами твірних доводиться, що виконуються властивості рівномірності і однорідності. Це дозволяє для знаходження діаметра застосовувати однорідний алгоритм пошуку вниз. При застосувані були отримані точні значення діаметрів знакозмінних груп для перших 43 степенів.

Ключові слова і фрази: граф Келі, діаметр графа, система твірних, знакозмінна група. 